\title{
The Local Structure of Tilings and their Integer Group of Coinvariants
}

\author{
Johannes Kellendonk \\ Department of Mathematics, King's College London, \\ Strand, London WC2R 2LS \\ E-mail: johannes@mth.kcl.ac.uk
}

\begin{abstract}
The local structure of a tiling is described in terms of a multiplicative structure on its pattern classes. The groupoid associated to the tiling is derived from this structure and its integer group of coinvariants is defined. This group furnishes part of the $K_{0}$-group of the groupoid $C^{*}$-algebra for tilings which reduce to decorations of $\mathbb{Z}^{d}$. The group itself as well as the image of its state is computed for substitution tilings in case the substitution is locally invertible and primitive. This yields in particular the set of possible gap labels predicted by $K$-theory for Schrödinger operators describing the particle motion in such a tiling.
\end{abstract}

KCL-TH-95-6 


\section{Introduction}

Finding topological objects to characterize or even classify tilings could be seen as a major motivation to study tilings. This article is less ambitious but already has the taste of it in that it is largely devoted to the study of such an object: the integer group of coinvariants associated to the tiling and the range of a state on it. But it takes its stimulation from two areas of application. One is the $K$-theoretical gap labelling of Schrödinger operators describing the motion of a particle in a tiling (and potentially many more features of solids for which a model by a tiling is appropriate), and the other concerns topological dynamical systems on the Cantor set. Let us briefly put these two areas into context.

1) Tilings furnish (discrete) models of solids which may not be periodic as for instance quasicrystals. A typical question arrising is that after the nature of the spectrum of a particle moving in the tiling (e.g. a phonon or an electron). The corresponding Schrödinger operator lies in the algebra of observables but its diagonalization is for non periodic tilings to difficult to carry out at present. To obtain at least some qualitative description of the spectrum Bellissard proposed the $K$-theoretical gap labelling [1]. This gap labelling requires the computation of a topological invariant of a $C^{*}$-algebra which may be taken to be the algebra of observables, namely of the range of a tracial state on its $K_{0}$-group. The algebra of observables coincides with the algebra associated to the tiling and the tracial state comes from a trace on it. The $K_{0}$-group contains the integer group of coinvariants (at least for a large class of tilings) and the range of the tracial state equals its range on the coinvariants. This range is a countable subgroup of $\mathbb{R}$ which contains the possible labels of gaps in the spectrum of a Schrödinger operator, i.e. its elements are the gap labels predicted by $K$-theory.

As it is determined by a topological invariant of the $C^{*}$-algebra associated to the tiling the $K$-theoretical gap labelling will be of use if the nature of the tiling is incorporated in the operator in a strong enough way so that all values of the above group (lying between 0 and 1) correspond to gaps. Although the quantification of the latter property is in general (in particular in higher dimensions) an unsolved problem we find it still worth persuing as there are no analytical alternatives in higher dimensions so far.

2) A certain class of tilings, the decorations of $\mathbb{Z}^{d}$, yield $d$-dimensional topological dynamical systems of the Cantor set. Such a topological dynamical system is given by $d$ commuting homeomorphisms, and a tiling which consists of decorated $d$-dimensional unit cubes touching nicely at their faces yields an example, the $d$ independent shifts yielding commuting homeomorphisms of the hull of the tiling. In one dimension the study of the $K$-group of the associated $C^{*}$-algebra (the one usually associated to the dynamical system coincides with the one associated to the tiling) turned out to be very fruitful. The ordered $K_{0}$-group with order unit, which coincides with the integer group of coinvariants as an unordered group, furnishes an invariant classifying the classes of strong orbit equivalent dynamical systems [2, 3]. In higher dimensions the interpretation of the $K$-group in terms of the properties of dynamical systems is yet less clear but it was recently realized that the (unordered) $K$-groups may be decomposed

into cohomology groups of the group $\mathbb{Z}^{d} \mathbb{4}$. In particular the group of coinvariants furnishes part of the $K_{0}$-group thus motivating its computation which is carried out 
below for tilings which allow for an invertible substitution. In turn, most of the analysis done for decorations of $\mathbb{Z}^{d}$ remains valid for a much larger class of tilings including the Penrose tilings, as will be shown below using the concept of reduction.

Although the above applications are formulated in the framework of $K$-theory of $C^{*}$ algebras we shall not emphasize the $C^{*}$-algebraic aspects in this article since the topological invariant discussed here may be defined purely on the level of spaces, i.e. here of groupoids. The article is organized as follows.

In the first section the groupoid associated to a tiling is described. We proceed along lines sligthly different from [5] by first introducing a multiplicative structure on the pattern classes of the tiling. This multiplicative structure is almost a groupoid, and using the notion of the radius of a pattern class one obtains the groupoid associated to the tiling in a way which makes its local nature transparent. In contrast to [5] this groupoid is only principal if the tiling is not periodic whereby we gain that it yields the right groupoid $C^{*}$-algebra in the periodic case, too. We then define the integer group of coinvariants associated to the tiling. Naturally, local manipulations, i.e. deformations of patterns, appear as maps which are close to being homomorphims of the introduced almost-groupoids. In fact, a substitution can be algebraically defined as a particular kind of such a homomorphism. We discuss the concept of reduction and the notion of a decoration of $\mathbb{Z}^{d}$ and show that many tilings occuring in the description of quasicrystals reduce to decorations of $\mathbb{Z}^{d}$.

Section 2 is devoted to the $C^{*}$-algebraic characterization and contains some details about the $K$-theoretical gap labelling. We first recall the definition of the algebra associated to the tiling. The main result needed concerns tilings which reduce to decorations of $\mathbb{Z}^{d}$ and states that their $C^{*}$-algebra is stably isomorphic to a crossed product with $\mathbb{Z}^{d}$. In particular the $K$-theory of such a tiling is that of a topological dynamical system.

The third section, which is kept independent of sections 1.4 and 2, is restricted to tilings which allow for an invertible substitution. Geometrically such a substitution may be obtained from an deflation but the analysis remains purely algebaic. The existence of an invertible substitution reflects in the possibility of obtaining an injective coding of the tiling in terms of the paths over a graph. This is technically the basis for the computation of the integer group of coinvariants and the range of its state. For one dimensional substitutions similar approaches have been successfully carried out, both, for the range of the state [6, 7] as well as for the group itself [8, 9]. In higher dimensions results regarding the range of the state have been obtained under the restriction that the substitution forces its border [5]. The results obtained here are independent of the dimension of the tiling, the only requirements being that the substitution is (locally) invertible and, for the determination of the range of the state, primitive. In fact, we obtain a family of codings and graphs for a tiling which for substitutions which force their border all coincide and have the substitution matrix as connectivity matrix but are more complicated in the general case. As applications we discuss the Thue-Morse substitution sequence and the Penrose tilings. 


\section{Tilings and groupoids}

Groupoids and almost-groupoids play a central role in the description of tilings. They carry a structure which generalizes a group structure in so far as the multiplication of two elements $x$ and $y$ is only defined provided $(x, y)$ lie in a specified set. We shall indicate this by $x \vdash y$. As a general reference to groupoids which includes groupoid$C^{*}$-algebras we use [10].

\subsection{Almost-groupoids}

Definition 1 An almost-groupoid is a set $\Gamma$ together with a subset $\Gamma^{\vdash} \subset \Gamma \times \Gamma$ of composable elements (we write $x \vdash y$ for $(x, y) \in \Gamma^{\vdash}$ ) and a product map $x \vdash y \mapsto x y$ : $\Gamma^{\vdash} \rightarrow \Gamma$ and an inversion map $x \mapsto x^{-1}: \Gamma \rightarrow \Gamma$, such that the following relations hold:

$$
\begin{aligned}
& \text { I1 }\left(x^{-1}\right)^{-1}=x, \\
& \text { I2 } x \vdash y \text { implies } y^{-1} \vdash x^{-1} \text {, in which case }(x y)^{-1}=y^{-1} x^{-1}, \\
& \text { I3 } x \vdash x^{-1} \text { and } x \vdash x^{-1} x \text { and } x x^{-1} x=x, \\
& \text { A }(x \vdash y \text { and } x y \vdash z) \text { whenever }(x \vdash y z \text { and } y \vdash z) \text {, in which case }(x y) z=x(y z) \text {. }
\end{aligned}
$$

The elements of $\Gamma^{0}=\left\{x^{-1} x \mid x \in \Gamma\right\}$ are called units. We will make frequent use of the maps $L, R: \Gamma \rightarrow \Gamma^{0}$ (often denoted $r, d$ ) defined by

$$
L(x)=x x^{-1} \quad R(x)=x^{-1} x .
$$

An almost-groupoid is called principal if the map $(L, R): \Gamma \rightarrow \Gamma^{0} \times \Gamma^{0}$ is injective. It defines orbits in the space of units: $u$ and $v$ are called orbit equivalent, $u \sim_{o} v$, whenever there is an $x$ such that $L(x)=u$ and $R(x)=v$. Note that $x \vdash y$ is equivalent to $x \vdash y y^{-1} y$ and $y y^{-1} \vdash y$ so that it is by $A$ equivalent to $x \vdash y y^{-1}$. In particular, $R(x)=L(y)$ implies $x \vdash y$.

Definition 2 A groupoid is an almost-groupoid for which cancelation holds, i.e.

$$
C x \vdash y \text { and } x \vdash z \text { imply } y=z .
$$

This definition of a groupoid is equivalent to the usual one as e.g. presented in [10]. It is not difficult to show that the cancelation axiom $\mathrm{C}$ indeed implies that

- $x \vdash y$ implies $R(x)=L(y)$,

- $x \vdash y$ and $y \vdash z$ imply $x \vdash z$.

In particular the above implications do not have to hold for almost-groupoids and associativity is only guaranteed provided all multiplications are defined. The lack of cancelation may be put into an order.

Definition 3 The order of an almost-groupoid is defined by

$$
x \preceq y \quad \text { iff } \quad x \vdash y^{-1} \quad \text { and } \quad x y^{-1}=y y^{-1} .
$$


A topological almost-groupoid is an almost-groupoid which carries a topology such that the product and the inversion map are continuous, $\Gamma^{\vdash}$ carrying the relative topology. A (locally compact) groupoid is called $r$-discrete if $\Gamma^{0}$ is open.

A homomorphism of almost-groupoids is a map which maps composable elements resp. units onto composable elements resp. units and preserves multiplication and inversion. In particular it preserves the order. A homomorphism of topological almost-groupoids is a continuous homomorphism of almost-groupoids.

Definition 4 An inverse semi-group is an almost-groupoid for which $\Gamma^{\vdash}=\Gamma \times \Gamma$.

Any almost-groupoid can be made into an inverse semi-group by introducing an extra element 0 and extending the multiplication to any $(x, y) \in \Gamma \times \Gamma$ by

$$
x y= \begin{cases}x y & \text { if } x \vdash y \\ 0 & \text { else }\end{cases}
$$

and $0 x=x 0=0,00=0.0$ is its own inverse and greater than any other element. Conversely we could see an almost-groupoid as an inverse semi-group with an element 0 satisfying $0 x=x 0=0$ for all $x$. However, homomorphisms of almost-groupoids may in general not be extended to homomorphisms of inverse semi-groups and vice versa. The difference which makes homomorphisms of inverse semi groups unsuitable for the purposes of this article is that for a homomorphism $\varphi$ of an almost-groupoid, even if $\varphi(x) \vdash \varphi(y)$, it has only to satisfy $\varphi(x) \varphi(y)=\varphi(x y)$ if $x \vdash y$.

Examples: 1) The principal groupoid which will be associated to a tiling is given by an equivalence relation, i.e. its elements are pairs of equivalent elements of some set $X$. Multiplication is only defined for pairs $(x, y),\left(x^{\prime}, y^{\prime}\right)$ if $x^{\prime}=y$ and then given by $(x, y)(y, z)=(x, z)$, and inversion by $(x, y)=(y, x)^{-1}$. The topology of the groupoids in question need in general not to coincide with the relative topology from $X \times X$.

2) Sometimes the equivalence may be expressed as orbit equivalence under the (right) action of a group $S$ acting on $X: x \sim y$ whenever $\exists s \in S: y=x \cdot s$. This leads to the consideration of another kind of groupoid which is called transformation group [10]. Its space is the Cartesian product of $X$ with $S$ (here always considered to carry the product topology) and the groupoid structure is defined by $(x, s)\left(x^{\prime}, t\right)=(x, s t)$ provided $x^{\prime}=x \cdot s$ and $(x, s)^{-1}=\left(x \cdot s, s^{-1}\right)$. We write it as $X \times_{\alpha} S, \alpha$ indicating the action. It may be viewed as the groupoid defined by orbit equivalence only if $S$ acts freely on $X$.

3) Any groupoid $\Gamma$ defines an inverse semi-group $\mathcal{I S G}(\Gamma)$ consisting of so-called $\Gamma$-sets. A $\Gamma$-set is a subset of $\Gamma$ for which the restrictions of $L$ and $R$ to it are both injective. If $X, Y$ are such $\Gamma$-sets (which may be empty) then $X Y:=\{x y \mid x \in X, y \in Y, x \vdash y\}$ and $X^{-1}:=\left\{x^{-1} \mid x \in X\right\}$. This multiplication of sets is not only for $\Gamma$-sets defined. We will make frequent use of it.

\subsection{Tilings}

Borrowing the terminology for $d=2$ from [11] a $d$ dimensional tiling is a (countable) family of closed subsets of $\mathbb{R}^{d}$, called the tiles, which cover $\mathbb{R}^{d}$, overlap at most at 
their boundaries and may carry an additional decoration, e.g. to break symmetries. We restrict the possible shape of tiles through the requirements that they are of finite size, i.e they fit inside an $r$-ball for finite $r$, and they are the closure of their interior. We shall consider the geometrical objects as equivalence classes under translations in $\mathbb{R}^{d}$ (but not under rotations or reflections). A tiling or its class $T$ defines an almostgroupoid as follows.

Consider the set of pattern classes (with resp. to translation) of $T$ where a pattern of a tiling is given by a finite subset of its tiles. A pattern class $M$ together with two tiles chosen from it (pointed out) will be called a doubly pointed pattern class. It will be denoted by $M_{x y}$ where $x$ is the first and $y$ the second pointed tile. The set of all doubly pointed pattern classes of $T$ will be denoted by $\mathcal{M}_{\mathbb{I I}}(T)$ or simply by $\mathcal{M}_{\mathbb{I I}}$ if the tiling is clear from the context.

$\mathcal{M}_{\text {II }}$ carries an order structure: $M_{x_{1} x_{2}} \grave{\precsim} N_{y_{1} y_{2}}$ iff the pattern class of $N_{y_{1} y_{2}}$ contains the pattern class of $M_{x_{1} x_{2}}$ at such a position that tiles $x_{1}$ resp. $x_{2}$ become $y_{1}$ resp. $y_{2}$, c.f. figure.

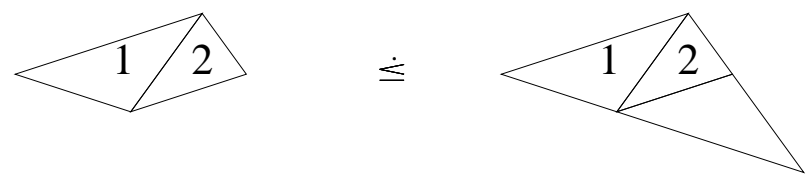

Moreover $\mathcal{M}_{\mathbb{I I}}$ carries the structure of an almost-groupoid whose order structure coincides with the one just introduced: Two elements $M_{x_{1} x_{2}}, N_{y_{1} y_{2}}$ are composable iff there is an $L_{z_{1} z_{2}}$ and a third tile $z$ of $L$ such that $M_{x_{1} x_{2}} \grave{\precsim} L_{z_{1} z}$ and $N_{y_{1} y_{2}} \grave{\precsim} L_{z z_{2}}$. We then define the product

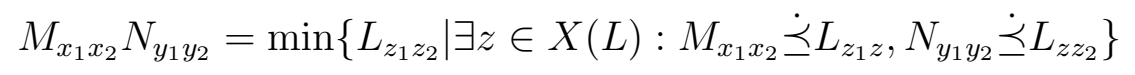

where the minimum is taken with respect to $\preceq$ and $X(L)$ denotes the tiles of the pattern class L. E.g.:
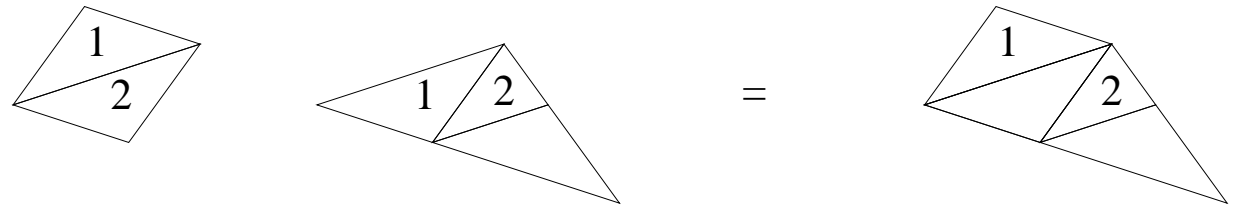

The inversion is given by

$$
\left(M_{x_{1} x_{2}}\right)^{-1}=M_{x_{2} x_{1}} .
$$

It is straightforward to see that $\preceq$ equals the order relation of the almost-groupoid. So we will leave the dot away. 
We consider $\mathcal{M}_{\mathbb{I}}$ as topological almost-groupoid carrying the discrete topology. The space of units $\mathcal{M}_{\mathbb{I}}{ }^{0}$ is given by the elements of the form $M_{x x}$ which we simply write $M_{x}$ and call pointed pattern classes. The maps $L$ and $R$ act as

$$
L\left(M_{x y}\right)=M_{x x} \quad R\left(M_{x y}\right)=M_{y y} .
$$

Let $X(T)$ be the set of tiles of $T$ where we identify tiles if they are mapped onto each other by a translation which is a multiple of a period of the tiling. (Since $T$ is an equivalence class we are forced to do this.) In particular, for tilings which are completely periodic, i.e. periodic in $d$ independent directions, $X(T)$ is a finite set. We call a tiling together with a chosen tile a pointed tiling and denote it by $T_{x}$. Let $M_{r}\left(T_{x}\right) \in \mathcal{M}_{\mathbb{I I}}^{0}$ be the smallest pointed pattern class which covers all balls of radius $r$ having their center inside tile $x$ and keep this tile chosen. Define $r_{c}\left(T_{x}, T_{x^{\prime}}\right):=\sup \left\{r \mid M_{r}\left(T_{x}\right)=M_{r}\left(T_{x^{\prime}}\right)\right\}$. Then $d\left(T_{x}, T_{x^{\prime}}\right):=e^{-r_{c}\left(T_{x}, T_{x^{\prime}}\right)}$ yields a metric on $\left\{T_{x} \mid x \in X(T)\right\}$ [5].

Definition 5 The hull $\Omega$ of $T$ is the completion of $\left\{T_{x} \mid x \in X(T)\right\}$ with respect to the tiling metric $d\left(T_{x}, T_{x^{\prime}}\right)=e^{-r_{c}\left(T_{x}, T_{x^{\prime}}\right)}$.

Note that $\Omega$ is a finite set if and only if $T$ is completely periodic. The elements of $\Omega$ may be interpreted as pointed tilings since any of their patterns is eventually determined by some $T_{x}$. In other words $\omega \in \Omega$ may be thought of as being approximated by an increasing chain of pointed pattern classes (which we now write shorter $u$ ).

Definition 6 A sequence of pointed pattern classes $\left\{u_{\nu}\right\}_{\nu}$ is called approximating if

1. any finite number of elements is composable, i.e. $\forall k \geq 1: u_{1} \ldots u_{k} \vdash u_{k+1}$,

2. $\operatorname{rad}\left(u_{\nu}\right) \rightarrow \infty$ where $\operatorname{rad}(u)$ is the largest $r$ such that all balls of radius $r$ having their center inside the pointed tile are covered by $u$.

An approximating sequence approximates a unique pointed tiling which we write as its limit, namely $\omega=\lim u_{\nu}$ is the pointed tiling which contains all pointed patterns $u_{\nu}$ at its pointed tile. It is clear that any pointed tiling has an approximation (just choose a divergent sequence $\left\{r_{\nu}\right\}_{\nu}$ in $\mathbb{R}^{+}$and take $\left.M_{r_{\nu}}(\omega)\right)$ so that we may carry over the notion $\preceq$ by writing $u \preceq \omega$ if $u \preceq u_{\nu}$ for some element of an approximation of $\omega$. The pattern classes of $\omega$ are pattern classes of $T$ as well, i.e. for all $\omega \in \Omega, \mathcal{M}_{\mathbb{I}}(\omega) \subset \mathcal{M}_{\mathbb{I}}$. One says that $\omega$ is locally homomorphic to $T_{x}$ (or $T$ ). Consequently $\omega$ (or its class) is called locally isomorphic to $\omega^{\prime}$ if $\mathcal{M}_{\mathbb{I}}(\omega)=\mathcal{M}_{\mathbb{I}}\left(\omega^{\prime}\right)$. $T$ is called minimal if it is locally isomorphic to any $\omega \in \Omega$ which is equivalent to saying that the closure of the orbit of any $\omega$ equals $\Omega$.

Since the range of $d\left(T_{x}, \cdot\right)$ is discrete away from 0 the hull is completely disconnected (zero dimensional). This means that the topology is generated by sets which are both open and closed. In fact, to any unit $u \in \mathcal{M}_{\mathbb{I}}{ }^{0}$ we may assign an open and closed subset

$$
U_{u}:=\{\omega \in \Omega \mid u \preceq \omega\}
$$

of $\Omega$. The collection of all of these yields a basis for the topology.

Let $\mathcal{M}_{\mathbb{I I} 2, \neq}$ denote the set of all doubly pointed pattern classes which are composed of two neighbored tiles (i.e. which have boundaries with nonzero intersection) and 
such that the first pointed tile is not equal to the second. $\mathcal{M}_{\mathbb{I} 2, \neq}$ generates $\mathcal{M}_{\mathbb{I}} \backslash \mathcal{M}_{\mathbb{I I} 1}$ where $\mathcal{M}_{\mathbb{I} 1}$ are the pointed pattern classes consisting of one tile only. The following compactness condition will be required furtheron.

- $\mathcal{M}_{\mathbb{I I} 2, \neq}$ is a finite set.

Under this condition $\Omega$ is compact. In fact, this condition is equivalent to the requirement that for any $r>0$ there are only finitely many pointed pattern classes which are covered by a ball of radius $r$, and the latter has been used to prove compactness of $\Omega$ in [5].

To keep contact with [5] where proofs of the above statements may be found, we visualize a (non periodic) pointed tiling $T_{x}$ as a particular representative of $T$ in $\mathbb{R}^{d}$. For this we choose a reference point 0 in $\mathbb{R}^{d}$ and introduce a puncture for each pattern class of a tile (i.e. the puncture is a point of the tile) so that $T_{x}$ is the representative for which tile $x$ lies with its puncture on 0 .

The almost-groupoid $\mathcal{M}_{\mathbb{I I}}$ acts on $\Omega$ from the right (and analogously from the left). Let

$$
\Omega^{\triangleright}=\{(\omega, c) \mid L(c) \preceq \omega\} \subset \Omega \times \mathcal{M}_{\mathbb{I I}}
$$

with relative topology. Define $\gamma: \Omega^{\triangleright} \rightarrow \Omega:(\omega, c) \mapsto \omega \cdot c$ through an approximating sequence: Let $\lim u_{\nu}=\omega$ and set

$$
\left(\lim u_{\nu}\right) \cdot c:=\lim R\left(u_{\nu} c\right) .
$$

More pictorially with $\omega=T_{x}^{\prime}$ and $M_{y} \preceq T_{x}^{\prime}$ we have $T_{x}^{\prime} \cdot M_{y y^{\prime}}:=T_{x^{\prime}}^{\prime}$ where $x^{\prime}$ is the tile of $T^{\prime}$ which coincides with $y^{\prime}$ once $M_{y}$ has been realized as the pattern at $x$ in $T^{\prime}$. Note that

$$
L(c) \preceq \omega \text { and } L\left(c^{\prime}\right) \preceq \omega \cdot c \quad \Longleftrightarrow \quad c \vdash c^{\prime} \text { and } L\left(c c^{\prime}\right) \preceq \omega
$$

and that in this case

$$
\gamma\left(\gamma(\omega, c), c^{\prime}\right)=\gamma\left(\omega, c c^{\prime}\right)
$$

Moreover $\gamma\left(\cdot, M_{x y}\right): U_{M_{x}} \rightarrow U_{M_{y}}$ is continuous so that we might call it a continuous right action of an almost-groupoid. Let us define an equivalence relation on $\Omega^{\triangleright}$ using approximations for the elements of $\Omega$ :

$$
\left(\lim u_{\nu}, c\right) \sim\left(\lim u_{\nu}, c^{\prime}\right) \quad \text { whenever } \exists n: u_{n} c=u_{n} c^{\prime} .
$$

It is straightforward to see that this definition is independent of the approximation and that the relation is transitive. We denote the equivalence classes by $[\omega, c]$. These classes may be visualized as doubly pointed tilings, namely $\left[T_{x}^{\prime}, M_{y z}\right]$ can be represented by $T_{x z}^{\prime}$, z being the tile of $T_{x}^{\prime}$ corresponding to the $z$ in $M_{y z}$ once the latter has been identified in $T_{x}^{\prime}$ such that $y$ coincides with $x$.

Lemma 1 Let $\mathcal{R}$ be quotient of $\Omega^{\triangleright}$ by the above equivalence relation with quotient topology and consider the groupoid structure defined by

$$
[\omega, c]\left[\omega^{\prime}, c^{\prime}\right]=\left[\omega, c c^{\prime}\right] \quad \text { provided } \omega^{\prime}=\omega \cdot c
$$


and

$$
[\omega, c]^{-1}=\left[\omega \cdot c, c^{-1}\right] .
$$

Then $\mathcal{R}$ is an $r$-disrete groupoid. Its space of units is homeomorphic to $\Omega$.

Proof: First, note that (13) is well defined because of (10,11). It is straightforward to check that $\mathcal{R}$ satisfies the axioms of a groupoid. The topology of $\mathcal{R}$ is generated by sets of the form

$$
\mathcal{U}_{c}:=\left[U_{L(c)} \times\{c\}\right]=\{[\omega, c] \mid L(c) \preceq \omega\} .
$$

$\mathcal{U}_{c}^{-1}=\mathcal{U}_{c^{-1}}$ shows continuity of the inversion and $m^{-1}\left(\mathcal{U}_{c}\right)=\bigcup_{c_{1} \vdash c_{2}, c \prec c_{1} c_{2}} \mathcal{U}_{c_{1}} \times \mathcal{U}_{c_{2}}$ that of multiplication $m: \mathcal{R}^{\vdash} \rightarrow \mathcal{R}$. The space of units is $\{[\omega, u] \mid u=L(u) \preceq \omega\}$ which is open and homeomorphic to $\Omega$ via $[\omega, u] \mapsto \omega$.

Definition 7 We call the groupoid $\mathcal{R}$ of Lemma 1 the groupoid associated to $T$.

The orbit of $\mathcal{R}$ through $\omega$ is given by $[\omega]_{o}=\{\omega \cdot c \mid L(c) \preceq \omega\}$ or more pictorial $\left[T_{x}^{\prime}\right]_{o}=\left\{T_{x}^{\prime} \mid x \in X\left(T^{\prime}\right)\right\}$. Hence $\left[T_{x}^{\prime}\right]_{o}$ is the equivalence class under translation $T^{\prime}$ : $T_{x} \sim_{o} T_{y}^{\prime}$ whenever $T=T^{\prime}$. If $T$ is not periodic then the quotient topology on the space of orbits $[\Omega]_{o}$ is not Hausdorff. Such a space is an example of a non commutative space in the sense of Connes 12. But the reader should be warned that what is called the non commutative space of Penrose tilings in [12] does not agree with the space of orbits of the hull of a Penrose tiling but is rather a quotient of it. We shall comment on that in section 3.5.2.

Let $\tilde{\gamma}: \mathcal{R} \rightarrow \Omega \times \Omega$ be given by $\tilde{\gamma}[\omega, c]=(\omega, \omega \cdot c)$.

Definition 8 The principal groupoid $\mathcal{S}$ associated to $T$ is the image of $\mathcal{R}$ under $\tilde{\gamma}$ with weak topology and groupoid structure defined by the orbit equivalence under $\mathcal{R}$.

Lemma $2 \mathcal{S}$ is isomorphic to $\mathcal{R}$ if and only if $T$ is non periodic.

Proof: Clearly $\tilde{\gamma}$ is a surjective and open homomorphism of topological groupoids. But it is only injective if $\gamma$ is free in the sense that $\omega \cdot c=\omega$ implies that $c$ is a unit. This is precisely the case if $T$ is not invariant under a translation.

In particular $\mathcal{R}$ is principal if $T$ has no periodicity. Note that the topology of $\mathcal{S}$ does not coincide with the relative topology inherited from $\Omega \times \Omega$. Its topology is generated by

$$
U_{M_{x y}}:=\tilde{\gamma}\left[\mathcal{U}_{M_{x y}}\right]=\left\{\left(T_{x^{\prime}}^{\prime}, T_{y^{\prime}}^{\prime}\right) \mid M_{x} \preceq T_{x^{\prime}}^{\prime}, T_{y^{\prime}}^{\prime}=T_{x^{\prime}}^{\prime} \cdot M_{x y}\right\} .
$$

In 55 the principal groupoid of a tiling was used instead of $\mathcal{R}$ but it turned out that from the point of view of physics the groupoid- $C^{*}$-algebra of $\mathcal{S}$ is not appropriate for tilings which have a periodicity in that it does not contain all translation operators in that case. 


\subsubsection{The integer group of coinvariants associated to a tiling}

An important ingredient for the characterization of a tiling is the integer group of coinvariants of its associated groupoid. This notion is borrowed from the case of transformation groups $X \times{ }_{\alpha} S$ where the action of $S$ on $X$ gives rise to an action on the group $C(X, \mathbb{Z})$ of continuous integer-valued functions, $\alpha(s)^{*}(f)(x)=f(x \cdot s)$. The coinvariants are then the quotient of $C(X, \mathbb{Z})$ by the subgroup generated by $\left\{f-\alpha(s)^{*}(f) \mid f \in C(X, \mathbb{Z}), s \in S\right\}$.

The ample semi group $\mathcal{A S G}(\Gamma)$ of an $r$-discrete groupoid $\Gamma$ is the sub-inverse-semigroup of $\mathcal{I S G}(\Gamma)$ given by its compact open $\Gamma$-sets. $\mathcal{A S G}(\Gamma)$ acts on $\mathcal{A S G}(\Gamma)^{0}$ by conjugation, $U \cdot \mathcal{U}:=\mathcal{U}^{-1} U \mathcal{U}$, leading to a partially defined action on $\Gamma^{0} \llbracket 10$ : for $u \in L(\mathcal{U})$,

$$
\{u\} \cdot \mathcal{U}=\mathcal{U}^{-1}\{u\} \mathcal{U}=\left\{c^{-1} u c\right\}
$$

where $c=L^{-1}(u)$. The integer group of coinvariants of $\Gamma$ (or group of coinvariants with coefficients in $\mathbb{Z}$ ) shall be the coinvariants of the $\mathbb{Z}$-module $C_{c}\left(\Gamma^{0}, \mathbb{Z}\right)$ of integer valued continuous functions over $\Gamma^{0}$ with compact support with respect to the above partially defined action of $\mathcal{A S G}(\Gamma)$. More precisely define $\eta: \mathcal{A S G}(\Gamma) \rightarrow C_{c}\left(\Gamma^{0}, \mathbb{Z}\right)$ by

$$
\eta(\mathcal{U}):=\chi_{L(\mathcal{U})}-\chi_{R(\mathcal{U})}
$$

where $\chi_{U}$ is the characteristic function on $U$ and let $E_{\Gamma}$ be the subgroup of $C_{c}\left(\Gamma^{0}, \mathbb{Z}\right)$ generated by im $\eta$.

Definition 9 The integer group of coinvariants of an $r$-discrete groupoid $\Gamma$ is

$$
H(\Gamma)=C_{c}\left(\Gamma^{0}, \mathbb{Z}\right) / E_{\Gamma} .
$$

If $\mathcal{U}, \mathcal{U}^{\prime}$ are disjoint $\Gamma$-sets and $\mathcal{U} \cup \mathcal{U}^{\prime}$ is a $\Gamma$-set as well then $\eta\left(\mathcal{U} \cup \mathcal{U}^{\prime}\right)=\eta(\mathcal{U})+\eta\left(\mathcal{U}^{\prime}\right)$. For transformation groups $\mathcal{A S G}\left(X \times{ }_{\alpha} S\right)$ contains disjoint unions of sets of the form $U \times\{s\}$ where $s \in S$ and $U \subset X$ is open and compact. Since $\eta(U \times\{s\})=\chi_{U}-\chi_{U \cdot s}$ the above definition is indeed a generalization.

Let us consider groupoids associated to tilings. Since the restrictions of $L$ and of $R$ to the sets $\mathcal{U}_{c}$ generating the topology are both injective $\mathcal{A S G}(\mathcal{R})$ contains unions of these sets. These unions may be chosen to be disjoined because the hull is zero dimensional. Now using $L\left(\mathcal{U}_{c}\right)=\mathcal{U}_{L(c)}$ one obtains

$$
H(\mathcal{R})=\left\langle\left\{\chi_{U_{u}} \mid u \in \mathcal{M}_{\mathbb{I}}^{0}\right\}\right\rangle /\left\langle\left\{\chi_{U_{L(c)}}-\chi_{U_{R(c)}} \mid c \in \mathcal{M}_{\mathbb{I}}\right\}\right\rangle .
$$

We call $H(\mathcal{R})$ also the integer group of coinvariants associated to $T$.

\subsection{Reduction}

The concept of reduction is very important in the sequel. In the context of dynamical systems it is sometimes called induction but we prefer the notion established for groupoids [13]. 
Let $\mathcal{N}$ be a sub-almost-groupoid of $\mathcal{M}_{\mathbb{I}}$ and define

$$
\Omega_{\mathcal{N}}:=\bigcup_{c \in \mathcal{N}} U_{R(c)}
$$

as well as

$$
\Omega_{\mathcal{N}}^{\triangleright}:=\Omega^{\triangleright} \cap\left(\Omega_{\mathcal{N}} \times \mathcal{N}\right)
$$

with relative topologies. We shall often consider the case in which $\mathcal{N}$ is finitely generated, i.e. $\mathcal{N}=\langle\mathcal{C}\rangle$ for a finite subset $\mathcal{C}$ of $\mathcal{M}_{\mathbb{I}}$, then $\Omega_{\mathcal{N}}=\bigcup_{c \in \mathcal{C}}\left(U_{R(c)} \cup U_{L(c)}\right)$. In fact, since $\Omega$ is compact and the $U_{u}$ form a basis of its topology any open and closed subset of $\Omega$ arrises in this manner with a finitely generated $\mathcal{N}$. There are two groupoids which naturally arrise from such a situation, one is the so-called reduction of $\mathcal{R}$ by $\Omega_{\mathcal{N}}$ :

$$
\mathcal{R}_{\Omega_{\mathcal{N}}}:=\left\{[\omega, c] \in \mathcal{R} \mid \omega, \omega \cdot c \in \Omega_{\mathcal{N}}\right\}
$$

(with relative topology), and the other is

$$
\mathcal{R}_{\mathcal{N}}:=\left[\Omega_{\mathcal{N}}^{\triangleright}\right]_{\mathcal{N}}
$$

where $[\omega, c]_{\mathcal{N}}=\left\{\left(\omega, c^{\prime}\right) \in[\omega, c] \mid c^{\prime} \in \mathcal{N}\right\}$ (with quotient topology).

Definition 10 We call $\mathcal{N}$ approximating if any $\omega \in \Omega_{\mathcal{N}}$ is approximated by a sequence $\left\{u_{\nu}\right\}_{\nu}$ with elements in $\mathcal{N}$ and generating if $[\omega, c]_{\mathcal{N}} \mapsto[\omega, c]: \mathcal{R}_{\mathcal{N}} \rightarrow \mathcal{R}_{\Omega_{\mathcal{N}}}$ is an isomorphism. We call $\mathcal{N}$ regular if any orbit of $\mathcal{R}$ intersects $\Omega_{\mathcal{N}}$, i.e. if $[\mathcal{R}]_{o}=\left[\mathcal{R}_{\mathcal{N}}\right]_{o}$.

Note that $[\omega, c]_{\mathcal{N}} \mapsto[\omega, c]: \mathcal{R}_{\mathcal{N}} \rightarrow \mathcal{R}_{\Omega_{\mathcal{N}}}$ provides an isomorphism if it is surjective. Note also that for minimal $T$ any non-empty $\mathcal{C} \in \mathcal{M}_{\text {II }}$ generates a regular sub-almostgroupoid $\langle\mathcal{C}\rangle$. None of the properties of the definition implies any other as counterexamples show. However, if $\mathcal{N}$ is generating then $\tilde{\mathcal{N}}=\left\{c_{1} u c_{2} \mid c_{i} \in \mathcal{N}, c_{1} \vdash c_{2}, R\left(c_{1}\right), L\left(c_{2}\right) \preceq\right.$ $\left.u \in \mathcal{M}_{\mathbb{I I}}^{0}\right\}$ is approximating and $\mathcal{R}_{\mathcal{N}}=\mathcal{R}_{\tilde{\mathcal{N}}}$.

Lemma 3 If $\mathcal{N}$ is approximating then the topology of $\Omega_{\mathcal{N}}$ is generated by sets of the form $U_{u}$ with $u \in \mathcal{N}^{0}$.

Proof: It is clear that the topology of $\Omega_{\mathcal{N}}$ is generated by sets of the form $U_{u v}$ where $u \in \mathcal{N}^{0}$ and $u \vdash v \in \mathcal{M}_{\mathbb{I}}{ }^{0}$. If $\mathcal{N}$ is approximating then there exists for any $\omega \in U_{u v}$ a $u_{\omega} \in \mathcal{N}^{0}$ such that $u v \preceq u_{\omega} \preceq \omega$. Hence $U_{u v}=\bigcup_{u v \preceq \omega} U_{u_{\omega}}$.

Lemma 4 If $\mathcal{N}$ is regular then for any $u \in \mathcal{M}_{\mathbb{I}}^{0}$ there is a finite subset $\left\{c_{i}\right\}_{i} \subset \mathcal{M}_{\mathbb{I}}$ with $U_{u}=\dot{\cup}_{i} U_{L\left(c_{i}\right)}$ and $U_{R\left(c_{i}\right)} \subset \Omega_{\mathcal{N}}$.

Proof: By regularity we may find for $\omega \in U_{u}$ a $c \in \mathcal{M}_{\text {II }}$ such that $\omega \cdot c \in \Omega_{\mathcal{N}}$. Let $r=\sup _{\omega \in U_{u}} \inf \left\{r^{\prime} \mid u \preceq M_{r^{\prime}}(\omega), \exists c \in \mathcal{M}_{\mathbb{I I}}: \omega \cdot c \in \Omega_{\mathcal{N}}, L(c) \preceq M_{r^{\prime}}(\omega)\right\}$. Since $U_{u}$ is compact $r$ is finite. Choose for any $\omega \in U_{u}$ a $c_{\omega}$ so that $\omega \cdot c_{\omega} \in \Omega_{\mathcal{N}}$ and $L\left(c_{\omega}\right)=M_{r}(\omega)$. In particular $U_{R\left(c_{\omega}\right)} \subset \Omega_{\mathcal{N}}$ and $\omega \in U_{L\left(c_{\omega}\right)} \subset U_{u}$. Hence $U_{u}=\bigcup_{u \preceq \omega} U_{L\left(c_{\omega}\right)}$. Since $U_{M_{r}(\omega)} \cap U_{M_{r}\left(\omega^{\prime}\right)}$ is either $U_{M_{r}(\omega)}$ or empty the union can be made disjoint and then, by compactness, finite.

The embedding $i: \mathcal{R}_{\Omega_{\mathcal{N}}} \hookrightarrow \mathcal{R}$ furnishes a surjective group homomorphism $i^{*}: C(\Omega, \mathbb{Z}) \rightarrow$ $C\left(\Omega_{\mathcal{N}}, \mathbb{Z}\right)$ which satisfies $i^{*} \circ \eta=\eta \circ i^{-1}$. Therefore $E_{\mathcal{R}_{\Omega_{\mathcal{N}}}}=E_{\mathcal{R}} \cap C\left(\Omega_{\mathcal{N}}, \mathbb{Z}\right)$ and $i^{*}$ induces a surjective homomorphism $H(\mathcal{R}) \rightarrow H\left(\mathcal{R}_{\Omega_{\mathcal{N}}}\right)$. 
Lemma 5 If $\mathcal{N}$ is regular then $H(\mathcal{R})=H\left(\mathcal{R}_{\Omega_{\mathcal{N}}}\right)$.

Proof: We have to show that that for $u \in \mathcal{M}_{\mathbb{I}}^{0}$ there is a finite $\left\{u_{i}\right\}_{i} \subset \mathcal{M}_{\mathbb{I}}^{0}$ with $U_{u_{i}} \subset \Omega_{\mathcal{N}}$ and $\left[\chi_{u}\right]_{E_{\mathcal{R}}}=\sum_{i}\left[\chi_{u_{i}}\right]_{E_{\mathcal{R}}}$. This follows immediately from the foregoing lemma taking $u_{i}=R\left(c_{i}\right)$.

Remark: Lemma 4 implies even that the groupoids $\mathcal{R}$ and $\mathcal{R}_{\Omega_{\mathcal{N}}}$ are continuously similar: Let $\mathcal{C}$ be the collection of $c_{i}$ 's such that $\Omega=\dot{U}_{i} U_{L\left(c_{i}\right)}$ and $U_{R\left(c_{i}\right)} \subset \Omega_{\mathcal{N}}$. Define $\Omega \rightarrow \mathcal{C}$ : $\omega \mapsto c_{\omega}, c_{\omega}$ being the unique element satisfying $L\left(c_{\omega}\right) \preceq \omega$, and $\epsilon: \mathcal{R} \rightarrow \mathcal{R}_{\Omega_{\mathcal{N}}}$ :

$$
\epsilon[\omega, c]=\left[\omega \cdot c_{\omega}, c_{\omega}^{-1} c c_{\omega \cdot c}\right] .
$$

Clearly $\epsilon$ is continuous and $\epsilon \circ i=i d_{\mathcal{R}_{\Omega_{\mathcal{N}}}}$ for the embedding $i: \mathcal{R}_{\Omega_{\mathcal{N}}} \rightarrow \mathcal{R}$. But also $i \circ \epsilon$ is similar to the identity on $\mathcal{R}$, namely the map $\Theta: \Omega \rightarrow \mathcal{R}: \Theta(\omega)=\left[\omega, c_{\omega}\right]$ satifies $\Theta(L([\omega, c])) \epsilon([\omega, c])=[\omega, c] \Theta(R([\omega, c]))$. This is a continuous version of the theorem of Ramsay [13] guaranteeing that the continuous cohomology of $\mathcal{R}_{\Omega_{\mathcal{N}}}$ is isomorphic to that of $\mathcal{R}$ [10].

\subsection{Locally defined maps between tilings}

We want to formulate in the present framework the concept of locally deriving one tiling from another, a concept which has been introduced for tilings in [14]. Roughly speaking a local derivation amounts to a deformation of pattern classes, e.g. by adding or deleting or deforming boundaries or decorations of tiles. Such a deformation will be given by a certain class of maps from sub-almost-groupoids of $\mathcal{M}_{\mathbb{I I}}$ to the doubly pointed pattern classes $\mathcal{M}_{\mathbb{I I}}^{\prime}=\mathcal{M}_{\mathbb{I I}}\left(T^{\prime}\right)$ of the other tiling class $T^{\prime}$. The results will be partly needed in the third section.

Lemma 6 Let $\mathcal{N}$ be an approximating sub-almost-groupoid of $\mathcal{M}_{\mathbb{I I}}$ and $\hat{\varphi}: \mathcal{N} \rightarrow \mathcal{M}_{\mathbb{I}}^{\prime}$ be a map which preserves composability, commutes with the inverse map and satisfies

$1 \hat{\varphi}(c) \hat{\varphi}\left(c^{\prime}\right) \preceq \hat{\varphi}\left(c c^{\prime}\right)$

2 (growth condition) there is a $t>0$ such that $|\operatorname{rad}(\hat{\varphi}(u))-t \operatorname{rad}(u)|$ is a bounded function on $\mathcal{M}_{\mathbb{I}}{ }^{0}$.

Then

$$
\varphi\left[\lim u_{\nu}, c\right]_{\mathcal{N}}:=\left[\lim \hat{\varphi}\left(u_{\nu}\right), \hat{\varphi}(c)\right]
$$

defines a homomorphism $\varphi: \mathcal{R}_{\mathcal{N}} \rightarrow \mathcal{R}^{\prime}$.

Proof: Let us first show that $\hat{\varphi}$ preserves the order. $c \preceq c^{\prime}$ is by definition $c \vdash c^{\prime-1}$ and $c^{\prime}=c R\left(c^{\prime}\right)$. But then $\hat{\varphi}(c) \preceq \hat{\varphi}(c) \hat{\varphi}\left(R\left(c^{\prime}\right)\right) \preceq \hat{\varphi}\left(c^{\prime}\right)$. Now let $\left\{u_{\nu}\right\}_{\nu}$ be an approximating sequence. Then for any $1<k, u_{1} \ldots u_{k} \vdash u_{k+1}$. The first condition and preservation of composability immediatly implies that $\hat{\varphi}\left(u_{1}\right) \ldots \hat{\varphi}\left(u_{k}\right) \vdash \hat{\varphi}\left(u_{k+1}\right)$. By the growth condition the radius of $\left\{\hat{\varphi}\left(u_{\nu}\right)\right\}_{\nu}$ diverges so it is an approximating sequence as well. Hence for $\omega=\lim u_{\nu}, \varphi(\omega)=\lim \hat{\varphi}\left(u_{\nu}\right)$ is well defined and clearly continuous. As well is $\Omega_{\mathcal{N}} \times \mathcal{N} \rightarrow \Omega^{\prime} \times \mathcal{M}_{\mathbb{I I}}^{\prime}:(\omega, c) \mapsto(\varphi(\omega), \hat{\varphi}(c))$. Thus we are left to show that $\varphi$ 
induces a map on $\left[\Omega_{\mathcal{N}}^{\triangleright}\right]_{\mathcal{N}}$ and preserves the groupoid structure. (By definition of the topology its continuity is then guaranteed.) We have $\hat{\varphi}(L(c)) \succeq L(\hat{\varphi}(c))$ and hence $\hat{\varphi}\left(R\left(u_{\nu} c\right)\right) \succeq R\left(\hat{\varphi}\left(u_{\nu}\right) \hat{\varphi}(c)\right)$. Therefore does $L(c) \preceq \omega$ imply $\hat{\varphi}(L(c)) \preceq \varphi(\omega)$ and $\varphi(\omega \cdot c)=\lim \hat{\varphi}\left(R\left(u_{\nu} c\right)\right)=\lim R\left(\hat{\varphi}\left(u_{\nu}\right) \hat{\varphi}(c)\right)=\varphi(\omega) \cdot \hat{\varphi}(c)$. This shows that $\varphi\left(\Omega_{\mathcal{N}}^{\triangleright}\right) \subset$ $\Omega^{\prime \triangleright}$ and that $\varphi$ maps equivalent elements onto equivalent ones, because equivalence may be expressed as $(\omega, c) \sim\left(\omega, c^{\prime}\right)$ whenever $\exists c^{\prime \prime}: c, c^{\prime} \preceq c^{\prime \prime}$ and $L\left(c^{\prime \prime}\right) \preceq \omega$. That $\varphi$ commutes with inversion on the level of groupoids follows from the corresponding hypothesis on the level of the almost-groupoids. Finally, multiplication is preserved if $\left(\varphi(\omega), \hat{\varphi}(c) \hat{\varphi}\left(c^{\prime}\right)\right) \sim\left(\varphi(\omega), \hat{\varphi}\left(c c^{\prime}\right)\right)$. In view of the above characterization of the equivalence this follows from $\hat{\varphi}(c) \hat{\varphi}\left(c^{\prime}\right) \preceq \hat{\varphi}\left(c c^{\prime}\right)$.

We say that $\varphi: \mathcal{R}_{\mathcal{N}} \rightarrow \mathcal{R}^{\prime}$ is locally defined. The corresponding homomorphism on the principal groupoids is simply $(\varphi, \varphi): \mathcal{S}_{\mathcal{N}}:=\mathcal{S} \cap\left(\Omega_{\mathcal{N}} \times \Omega_{\mathcal{N}}\right) \rightarrow \mathcal{S}^{\prime}$. Details of the proof of the following theorem, which is constructive, will be used in section 3 .

Theorem 1 Let $\varphi: \mathcal{R} \rightarrow \mathcal{R}^{\prime}$ be a locally defined homomorphism. If $\varphi$ has a left inverse then this left inverse is locally defined.

Proof: Let $a \in \mathcal{M}_{\mathbb{I} 1}, \mathcal{M}_{\mathbb{I} 1}$ denoting the set of pointed pattern classes of tiles. Since $\varphi\left(U_{a}\right)$ is open and compact we may find a finite subset $\Phi(a)$ of $\mathcal{M}_{\mathrm{II}}^{\prime}$ satisfying

$$
\varphi\left(U_{a}\right)=\bigcup_{v \in \Phi(a)} U_{v}
$$

Let $E^{0}(u)=\left\{e \in \mathcal{M}_{\mathbb{I}} \mid R(e)=u\right\}$ and denote by $x(e)$ the first pointed tile of $e$. The expression $u=\prod_{e \in E^{0}(u)} e^{-1} x(e) e$ motivates to define

$$
\Phi^{e}(u):=\left\{\hat{\varphi}\left(e^{-1}\right)\right\} \Phi(x(e))\{\hat{\varphi}(e)\}
$$

where we have used set multiplication, and

$$
\Phi(c):=\left(\prod_{e \in E^{0}(L(c))} \Phi^{e}(L(c))\right)\{\hat{\varphi}(c)\} .
$$

We claim that

$$
\varphi\left(\mathcal{U}_{c}\right)=\bigcup_{d \in \Phi(c)} \mathcal{U}_{d}
$$

Indeed, using that $u \preceq \omega$ whenever $\forall e \in E^{0}(u): x(e) \preceq \omega \cdot e^{-1}$ we get

$$
\begin{aligned}
\varphi\left(\mathcal{U}_{c}\right) & =\bigcap_{e \in E^{0}(L(c))}\left\{[\varphi(\omega), \hat{\varphi}(c)] \mid \omega \cdot e^{-1} \in U_{x(e)}\right\} \\
& =\left\{\left[\omega^{\prime}, \hat{\varphi}(c)\right] \mid \forall e \in E^{0}(L(c)): \omega^{\prime} \in \bigcup_{v \in \Phi(x(e))} U_{\left.\hat{\varphi}\left(e^{-1}\right) v \hat{\varphi}(e)\right\}}\right\} \\
& =\left\{\left[\omega^{\prime}, \hat{\varphi}(c)\right] \mid \omega^{\prime} \in \bigcup_{v \in \Phi(L(c))} U_{v}\right\}
\end{aligned}
$$


from which the claim follows. In particular, setting $\mathcal{N}=\Phi\left(\mathcal{M}_{\mathbb{I I}}\right)$, we have $\operatorname{im} \varphi=\mathcal{R}_{\mathcal{N}}^{\prime}$. Furthermore let $\Phi(c) \vdash \Phi\left(c^{\prime}\right)$, which shall mean that $\exists d \in \Phi(c) \exists d^{\prime} \in \Phi\left(c^{\prime}\right): d \vdash d^{\prime}$. Then from (29) we may conclude that $U_{R(c)} \cap U_{L(c)} \neq \emptyset$. Hence

$$
\Phi(c) \vdash \Phi\left(c^{\prime}\right) \text { implies } c \vdash c^{\prime} \text {. }
$$

Now set $W(d)=\left\{c \in \mathcal{M}_{\mathbb{I I}} \mid d \in \Phi(c)\right\}$ which by construction is finite. If $c, c^{\prime} \in W(d)$ then by (29) $\mathcal{U}_{c} \cap \mathcal{U}_{c^{\prime}} \neq \emptyset$ and hence $c$ and $c^{\prime}$ have a common greater element, e.g. $L(c) c^{\prime}$. Since $L(d) \in \Phi(L(c)) \cap \Phi\left(L\left(c^{\prime}\right)\right)$ also $L(d) \in \Phi\left(L(c) L\left(c^{\prime}\right)\right)$ so that $d \in \Phi\left(L(c) c^{\prime}\right)$. It follows that $\min \left\{c \mid \forall c^{\prime} \in W(d): c^{\prime} \preceq c\right\}=\max W(d)$. Define $\hat{\psi}: \mathcal{N} \rightarrow \mathcal{M}_{\mathbb{I I}}$ :

$$
\hat{\psi}(d):=\max W(d) \text {. }
$$

It commutes with the inverse. Because of $d \in \Phi(\hat{\psi}(d))$ and (30) $d \vdash d^{\prime}$ implies $\hat{\psi}(d) \vdash$ $\hat{\psi}\left(d^{\prime}\right)$. But it also implies $W(d) W\left(d^{\prime}\right) \subset W\left(d d^{\prime}\right)$ and hence $\hat{\psi}(d) \hat{\psi}\left(d^{\prime}\right) \preceq \hat{\psi}\left(d d^{\prime}\right)$.

Since $\Phi(A)$ is finite $\hat{\psi}$ and $\hat{\psi} \circ \hat{\varphi}$ satisfy the growth condition (with $t^{-1}$ resp. 1 in place of $t$ ) and $\mathcal{N}$ is approximating. Hence they extend to a homomorphisms $\mathcal{R}_{\mathcal{N}}^{\prime} \rightarrow \mathcal{R}$ resp. $\mathcal{R} \rightarrow \mathcal{R}$. Since $\psi \circ \varphi$ extends to the identity $\psi$ extends to a left inverse.

We say that $\varphi$ is locally invertible (on its image).

Lemma 7 If $T$ is non-periodic then the almost-groupoid $\mathcal{N}=\Phi\left(\mathcal{M}_{\mathbb{I I}}\right)$ occurring in the proof of the above theorem is generating.

Proof: Consider the commuting diagram

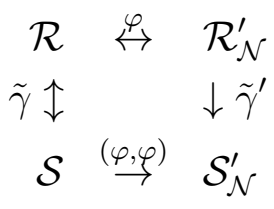

where $\tilde{\gamma}$ is an isomorphism since $T$ is non periodic. Clearly $(\varphi, \varphi)$ is injective. Suppose that it is not surjective. Then there must be an $x \sim_{o} y$ such that $\varphi^{-1}(x) \chi_{o} \varphi^{-1}(y)$. But this contradicts that $\varphi^{-1}$ is a left inverse which preserves (like any homomorphism of groupoids) orbits. Therefore $\tilde{\gamma}^{\prime}$ is bijective and hence $\mathcal{N}$ generating.

Counterexamples show that the requirement for $T$ to be non periodic is neccessary.

\subsection{Decorations of $\mathbb{Z}^{d}$}

For many tilings the groupoid is a transformation group the group being $\mathbb{Z}^{d}$. This is e.g. the case if the tiling is built from unit cubes which nicely touch at the faces. But since we allow for decorations these tilings do not have to be periodic. They are called decorations of $\mathbb{Z}^{d}$. In any case they allow for $d$ commuting homeomorphisms of the hull which are given by the $d$ independent shifts such that the orbits of $\mathcal{R}$ become orbits under the action of $\mathbb{Z}^{d}$ and $\mathcal{R}$ a transformation group $\Omega \times_{\alpha} \mathbb{Z}^{d}$. The structure of the $K$-groups of $C^{*}$-algebras defined by such transformation groups is fairly well understood and it turns out to be useful as well in the case where $\mathcal{R}$ merely has a reduction $\mathcal{R}_{\Omega_{\mathcal{N}}}$ which is isomorphic to $\Omega_{\mathcal{N}} \times{ }_{\alpha} \mathbb{Z}^{d}$. In view of Lemma 5 this already implies for regular $\mathcal{N}$ that the integer group of coinvariants coincide. 
Definition 11 We say that $T$ reduces to a decoration of $\mathbb{Z}^{d}$ if there is a regular $\mathcal{N}$ such that $\mathcal{R}_{\Omega_{\mathcal{N}}} \cong \Omega_{\mathcal{N}} \times{ }_{\alpha} \mathbb{Z}^{d}$. If $\mathcal{N}=\mathcal{M}_{\mathbb{I}}$ then $T$ is called a decoration of $\mathbb{Z}^{d}$.

Tilings which reduce to decorations of $\mathbb{Z}^{d}$ yield by definition $d$-dimensional topological dynamical systems. The topological space is $\Omega_{\mathcal{N}}$ which is homeomorphic to the Cantor set. These systems are topologically transitive and the notion of minimality in the sense of tilings and in the sense of dynamical systems coincide.

There is a large class of tilings which reduce to decorations of $\mathbb{Z}^{d}$. These tilings are composed of $d$ dimensional parallel epipeds which may be decorated. For their definition consider a set of $N$ vectors $\xi_{1}, \cdots, \xi_{N}$ which span $\mathbb{R}^{d}$. A set $J \subset\{1, \cdots, N\}$ containing $n$ elements, $n \leq d$, such that $\left\{\xi_{i}\right\}_{i \in J}$ are linear independent defines a subset $\left\{\sum_{i \in J} c_{i} \xi_{i} \mid c_{i} \in[0,1]\right\}$ of $\mathbb{R}^{d}$ which is an $n$ dimensional parallel epiped. We will call a translate of it an $n$-facet of type $J$. Their boundaries are $n^{\prime}$-facets, $n^{\prime}<n$, of type $J^{\prime} \subset J$. Let $T$ be a tiling of $\mathbb{R}^{d}$ consisting of possibly decorated $d$-facets which are arranged in such a way that

D1 the boundaries of tiles overlap, if at all, at common complete $d^{\prime}$-facets, $d^{\prime}<d$.

Provided the number of different decorations is finite, which we assume, there is only a finite number of pattern classes consisting of two tiles which touch each other so that the hull is compact.

If $d=N$ then $T$ itself a decoration of $\mathbb{Z}^{d}$ so here we are interested in $d<N$. Now fix a type $I_{0}$ of some tile appearing in $T$ and set

$$
\mathcal{C}=\left\{a \in \mathcal{M}_{\mathbb{I I} 1} \mid a \text { is of type } I_{0}\right\}
$$

where $\mathcal{M}_{\mathbb{I} 1}$ denotes the set of pointed pattern classes of tiles. For any $i \in I_{0}$ choose a (common) normal for $d$-1-facets of type $I_{0} \backslash\{i\}$, i.e. a positive and a negative side, and let $\mathcal{B}_{i} \subset \mathcal{M}_{\mathbb{I} 2, \neq}$ consist of those doubly pointed pattern classes which have as common boundary a $d$-1-facet of type $I_{0} \backslash\{i\}$ and such that their first resp. second pointed tile is on the positive resp. negative side of the common boundary. Then $\Omega_{\langle\mathcal{C}\rangle}=\bigcap_{i} \Omega_{\left\langle\mathcal{B}_{i}\right\rangle}$. Define $\breve{\alpha}_{i}: \Omega_{\left\langle\mathcal{B}_{i}\right\rangle} \rightarrow \Omega_{\left\langle\mathcal{B}_{i}\right\rangle}$ by

$$
\breve{\alpha}_{i}(\omega)=\omega \cdot c
$$

where $c$ is the unique element of $\mathcal{B}_{i}$ for which $L(c) \preceq \omega$. Clearly $\breve{\alpha}_{i}^{-1}(\omega)=\omega \cdot c^{-1}$ with the unique $c$ for which $R(c) \preceq \omega$. Now we require in addition that

D2 for all $i \in I_{0}$ and all $\omega \in \Omega_{\langle\mathcal{C}\rangle}$ there are natural numbers $n, m>0$ such that $\breve{\alpha}_{i}^{n}(\omega) \in \Omega_{\langle\mathcal{C}\rangle}$ and $\breve{\alpha}_{i}^{-m}(\omega) \in \Omega_{\langle\mathcal{C}\rangle}$.

Let $n_{i}^{ \pm}(\omega)$ be the smallest $n>0$ for which $\breve{\alpha}_{i}^{ \pm n}(\omega) \in \Omega_{\langle\mathcal{C}\rangle}$. This defines maps $n_{i}^{ \pm}$: $\Omega_{\langle\mathcal{C}\rangle} \rightarrow \mathbb{Z}^{>0}$ which are continuous by the compactness of $\Omega_{\langle\mathcal{C}\rangle}$. Consequently $\alpha$ : $\Omega_{\langle\mathcal{C}\rangle} \rightarrow \Omega_{\langle\mathcal{C}\rangle}$

$$
\alpha_{i}(\omega):=\breve{\alpha}_{i}^{n_{i}^{+}(\omega)}(\omega)
$$

are continuous maps (first return maps) having inverses $\alpha_{i}^{-1}:=\breve{\alpha}_{i}^{n_{i}^{-}}$. 
Lemma 8 Let $T$ be a d dimensional tiling which is composed of (possibly decorated) parallel epipeds and satisfies conditions $\mathrm{D} 1, \mathrm{D} 2$. Then the homeomorphisms $\alpha_{i}$ defined above commute pairwise. Moreover, $\langle\mathcal{C}\rangle$ defined by (39) is regular.

Proof: Draw in any tile which has $d$-1-facets of type $I_{0} \backslash\{i\}$ a line segment joining the middle points of the boundary facets of this type. (The middle point of an $n$-facet of type $J$ is at $\sum_{i \in J} \frac{1}{2} \xi_{i}$.) This segment goes through the middle point of the tile. All the line segments in a tiling fit together to yield a set of infinite lines in $\mathbb{R}^{d}$. Such a line will be called $i$-line its type being $i$. As an example the below figure shows a patch of an octogonal tiling with its line segments of two types. The four vectors $\left\{\xi_{1}, \ldots, \xi_{4}\right\}$ are given to the right, the lines are of type 1 and 3 .

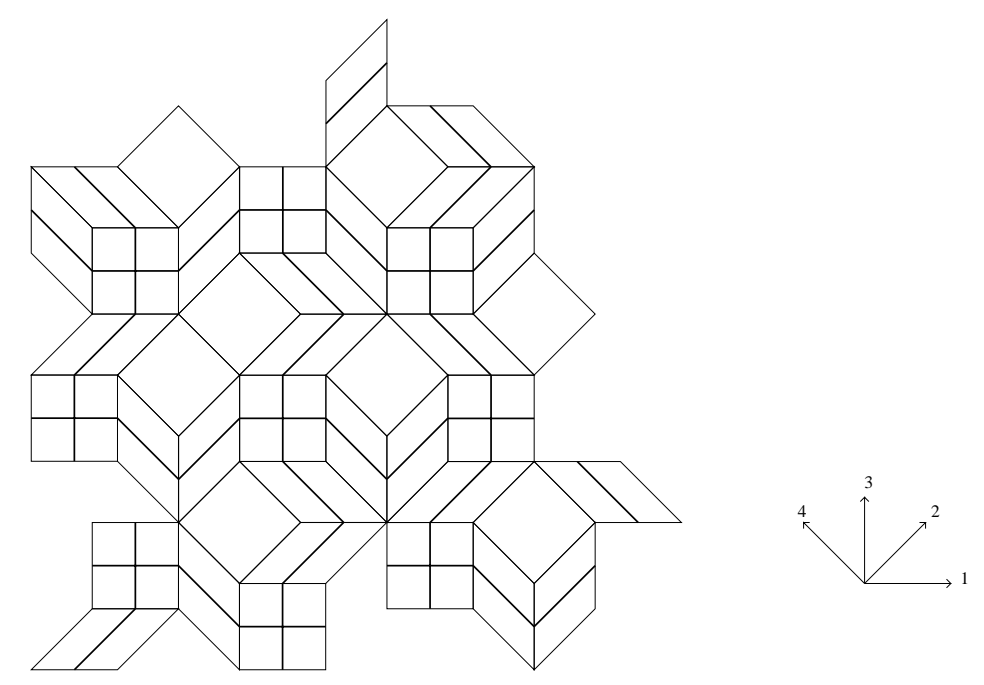

In general the lines have the properties:

1 If $l \neq l^{\prime}$ then $l \cap l^{\prime}$ contains at most one point (which is then the middle point of the tile through which they both go), and if $l$ and $l^{\prime}$ are of the same type then $l \cap l^{\prime}=\emptyset$.

2 Let $\pi_{i}$ be the orthogonal projection along the span of $\left\{\xi_{j}\right\}_{j \in I_{0} \backslash\{i\}}$. Then, for $d=2$ and any line which is of type $i, \pi_{i}(l)$ equals to the span of $\xi_{i}$.

Any type of line shall now be given the direction of the chosen normal of $I_{0} \backslash\{i\}$. The tiles belonging to it can be ordered and the application of $\breve{\alpha}_{i}$ can be geometrically interpreted as a shift to the next tile along an $i$-line. Condition D2 then reads that any line of type $i \in I_{0}$ contains in both directions infinitely many tiles of type $I_{0}$. Let us single out two elements of $I_{0}$ which we denote by 1 and 2 . Let $l_{0}, m_{0}$ be the lines of type 1 resp. 2 which go through $x$, the pointed tile of $T_{x} \in \Omega_{\langle\mathcal{C}\rangle}$. Let $m_{1}, l_{1}, m^{\prime}$ be the successor resp. of $m_{0}$ along $l_{0}$, of $l_{0}$ along $m_{0}$, and of $m_{0}$ along $l_{1}$, c.f. the below figure where this situation is indicated topologically. 


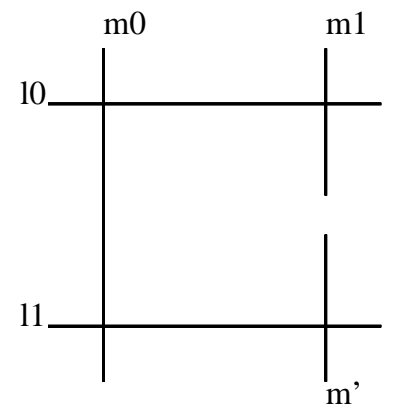

Then $\alpha_{1} \circ \alpha_{2}\left(T_{x}\right)=\alpha_{2} \circ \alpha_{1}\left(T_{x}\right)$ if and only if $m^{\prime}=m_{1}$.

We first proof that this is the case for $d=2$. By property 1 above $m^{\prime}$ can neither intersect $l_{1}$ at a second point nor $m_{0}$ at all nor can it intersect $l_{0}$ at a lower point than $m_{1}$ does. The same reasoning applies to $m_{1}$ so that, since $\pi_{2}\left(m^{\prime}\right)$ is the span of $\xi_{2}$, $m^{\prime} \cap m \neq \emptyset$. Hence $m^{\prime}=m_{1}$.

The case $d>2$ can be traced back to $d=2$ reducing $T_{x}$ to a two dimensional tiling $\overline{T_{x}}$ by the following process. Let $J_{0}=I_{0} \backslash\{1,2\}$. We define the $J_{0}$-neighborhood of a tile $a$ which has $d-2$-facets of type $J_{0}$ to consist of this tile an those tiles whose boundary intersects the boundary of $a$ at a $d$-2-facet of type $J_{0}$. Let $\overline{T_{x}}$ be the smallest connected component consisting of tiles having $d-2$-facets of type $J_{0}$ in such a way that, first, it contains with a tile its $J_{0}$-neighborhood, and second, it contains the pointed tile of $T_{x}$. In particular $\overline{T_{x}}$ is a connected topological manifold which contains complete 1and 2-lines. Let $\pi$ be the orthogonal projection along $\left\langle\left\{\xi_{i}\right\}_{i \in J_{0}}\right\rangle$. Then $\pi\left(\overline{T_{x}}\right)$ is a two dimenional tiling to which we can apply the above arguement leading to $\pi\left(m_{1}\right)=\pi\left(m^{\prime}\right)$. To see that this implies $m_{1}=m^{\prime}$ consider the homotopy $F_{t}\left(\xi_{i}\right)=(1-t) \xi_{i}+t \pi\left(\xi_{i}\right)$ for $i \notin J_{0}$ and $F_{t}\left(\xi_{i}\right)=\xi_{i}$ for $i \in J_{0} . F_{1}\left(\overline{T_{x}}\right)$ may be understood as the Cartesian product of $\pi\left(\overline{T_{x}}\right)$ with a $d$-2-facet of type $J_{0}$. Certainly no new intersections between lines in $F_{t}\left(\overline{T_{x}}\right)$ occurr for $t>0$ so that the decomposition shows that $\pi\left(m_{1}\right)=\pi\left(m^{\prime}\right)$ whenever $m_{1}=m^{\prime}$.

By compactness of $\Omega_{\langle\mathcal{C}\rangle}$ the distance of two neighbored lines has an upper and a lower bound. Hence for some $r$ any $u \in \mathcal{M}_{\mathbb{I}}{ }^{0}$ of radius larger than $r$ contains a tile of type $I_{0}$. Therefore $\langle\mathcal{C}\rangle$ is regular.

Theorem 2 Let $T$ be a d dimensional tiling which is composed of (possibly decorated) parallel epipeds and satisfies conditions D1,D2. Then it reduces to a $Z^{d}$ decoration.

Proof: The last lemma implies that the map $\Omega_{\langle\mathcal{C}\rangle} \times_{\alpha} \mathbb{Z}^{d} \rightarrow \mathcal{R}_{\Omega_{\langle\mathcal{C}\rangle}}:\left(\omega, e_{i}\right) \mapsto[\omega, c]$ where $c$ is uniquely determined by $c=c_{1} \ldots c_{n^{+}(\omega)}$ with $c_{k} \in \mathcal{B}_{i}, c_{k} \vdash c_{k+1}$, and $L(c) \preceq \omega$, is an isomorphism of groupoids.

All tilings which are obtained by the so-called generalized dual (or grid) method 15 fall under the vicinity of the above theorem provided they satisfy D2. In fact, the lines of different type can be understood objects dual to the hyperplanes of the grids so that 
we just inverted the construction of [15]. In particular all tilings obtained by the cut and projection method [16] (which is equivalent to the grid method using special grids only) reduce to decorations as they all satisfy D2.

Among the substitution tilings which reduce to decorations of $\mathbb{Z}^{2}$ are the AmmannBeenker- the Socolar- and the Penrose tilings.

\section{$2 \quad C^{*}$-algebraic characterization of tilings}

We review the $C^{*}$-algebraic constructions neccessary to formulate the $K$-theoretical gap labelling for particles moving in a tiling. As already mentionned the $K$-theoretical gap labelling may be seen as an invariant of a $C^{*}$-algebra. The natural candidate for this algebra is the algebra $A_{T}$ associated to the tiling $T$ in which the particle is moving since it contains all local operators involving translations and multiplications with pattern dependent functions.

Definition 12 The algebra $\mathcal{A}_{T}$ associated to a tiling $T$ is the reduced groupoid-C*algebra $C_{\text {red }}^{*}(\mathcal{R})$ of the groupoid $\mathcal{R}$ associated to $T$.

Any $r$-dicrete groupoid defines a reduced groupoid- $C^{*}$-algebra [10]. Specified to the groupoid $\mathcal{R}$ it is defined as follows: Let $C_{c}(\mathcal{R})$ be the $*$-algebra of continuous functions $f: \mathcal{R} \rightarrow \mathbb{C}$ with compact support and multiplication and involution given by

$$
\begin{aligned}
f * g[\omega, c] & =\sum_{\substack{\left[\omega \cdot c, c^{\prime}\right] \\
\omega, c \text { fixed }}} f\left[\omega, c c^{\prime}\right] g\left[\omega \cdot c c^{\prime}, c^{-1}\right], \\
f^{*}[\omega, c] & =\frac{f\left[\omega \cdot c, c^{-1}\right] .}{f[}
\end{aligned}
$$

$C_{c}(\mathcal{R})$ is generated by the characteristic functions $e_{c}$ onto $\mathcal{U}_{c}$ which satisfy the relations

$$
\begin{aligned}
e_{c} * e_{c^{\prime}} & = \begin{cases}e_{c c^{\prime}} & \text { if } c \vdash c^{\prime} \\
0 & \text { else }\end{cases} \\
e_{c}^{*} & =e_{c^{-1}} .
\end{aligned}
$$

Hence in any representation $e_{c}$ is represented as a partial isometry. $C_{\text {red }}^{*}(\mathcal{R})$ is the closure of $C_{c}(\mathcal{R})$ taken with respect to the reduced norm. This norm is defined using the family of representations $\pi_{[\omega]_{o}}$, one for each point in the non commutative space, acting on the Hilbert space of square summable functions $\Psi:[\omega]_{o} \rightarrow \mathbb{C}$ through

$$
\left(\pi_{[\omega]_{o}}\left(e_{c}\right) \Psi\right)(\omega)=\left\{\begin{array}{ll}
\Psi(\omega \cdot c) & \text { if } L(c) \preceq \omega \\
0 & \text { else }
\end{array} .\right.
$$

Now the reduced norm is given by $\|f\|_{\text {red }}=\sup _{[\omega]_{o} \in[\Omega]_{o}}\left\|\pi_{[\omega]_{o}}(f)\right\|$ where $\left\|\pi_{[\omega]_{o}}(f)\right\|$ is the operator norm. Since the topology of $\mathcal{R}$ has a countable basis $\mathcal{A}_{T}$ is separable.

As an example consider a tiling which is a decoration of $\mathbb{Z}^{d}$. That is there are $d$ commuting homeomorphisms $\alpha_{i}$ of the hull such that $\mathcal{R}=\Omega \times{ }_{\alpha} \mathbb{Z}^{d}$. To any continuous $f: \Omega \times_{\alpha} \mathbb{Z}^{d} \rightarrow \mathbb{C}$ with compact support one may assign the function $\hat{f}: \mathbb{Z}^{d} \rightarrow C(\Omega)$ 
through $\hat{f}(k)(\omega)=f(\omega, k)$. Carried over from (35).36) multiplication and involution then become convolution resp. involution twisted by $\alpha$ :

$$
\begin{aligned}
\hat{f} * \hat{g}(k) & =\sum_{m \in \mathbb{Z}^{d}} \hat{f}(m)(\hat{g}(k-m) \circ \alpha(m)) \\
\hat{f}^{*}(k) & =\frac{\hat{f}(-k) \circ \alpha(k)}{}
\end{aligned}
$$

The closure $C_{r e d}^{*}\left(\Omega \times_{\alpha} \mathbb{Z}^{d}\right)$ is isomorphic to $C(\Omega) \times_{\alpha} \mathbb{Z}^{d}$, the crossed product of $C(\Omega)$ with $\mathbb{Z}^{d}$ by the action $\alpha(k)(\hat{f}(m))=\hat{f}(m) \circ \alpha(k), \alpha(k)=\alpha_{1}^{k_{1}} \circ \ldots \circ \alpha_{d}^{k_{d}}$.

Remember that $T$ is minimal if the closure of the orbit of any $\omega$ equals $\Omega$. The lattice of closed (twosided) ideals of $\mathcal{A}_{T}$ may be identified with the lattice of open invariant subsets of $\Omega$, i.e. those open subsets which contain next to an element $T$ all its equivalent elements [10]. The groupoid $\mathcal{R}$ is called minimal if $\Omega$ does not contain any proper invariant open subset. But this is just excluded for minimal tilings. So if $T$ is minimal then $\mathcal{R}$ is minimal which implies that $\mathcal{A}_{T}$ is simple [10].

Lemma 5 has a $C^{*}$-algebraic counterpart. Two $C^{*}$-algebras $\mathcal{A}$ and $\mathcal{B}$ are called stably isomorphic if $\mathcal{A} \otimes \mathcal{K}$ is isomorphic to $\mathcal{B} \otimes \mathcal{K}$, where $\mathcal{K}$ is the algebra of compact operators (on an infinite dimensional separable Hilbert space). A theorem of Brown [17 states that a full reduction $\mathcal{B}$ of $\mathcal{A}$ is stably isomorphic to $\mathcal{A}$. In this context a reduction of $\mathcal{A}$ is determined by a projection $p \in \mathcal{A}$, namely given by $\mathcal{A}_{p}:=\{x \in \mathcal{A} \mid p x=x p=x\}$, and $\mathcal{A}_{p}$ is full if the twosided ideal generated by $p$ is dense in $\mathcal{A}$.

Lemma 9 Let $\mathcal{N} \subset \mathcal{M}_{\text {II }}$ be regular. Then $C_{\text {red }}^{*}\left(\mathcal{R}_{\Omega_{\mathcal{N}}}\right)$ is stably isomorphic to $\mathcal{A}_{T}$.

Proof: Let $\chi_{\Omega_{\mathcal{N}}}$ be the projection in $\mathcal{A}_{T}$ which is the characteristic function onto $\Omega_{\mathcal{N}}$ and $f \in C_{c}(\mathcal{R})$. Then $f * \chi_{\Omega_{\mathcal{N}}}=\chi_{\Omega_{\mathcal{N}}} * f=f$ if and only if supp $f \subset \mathcal{R}_{\Omega_{\mathcal{N}}}$. Hence $C_{c}\left(\mathcal{R}_{\Omega_{\mathcal{N}}}\right)$ is a dense subalgebra of $\left(\mathcal{A}_{T}\right)_{\chi_{\Omega_{\mathcal{N}}}}$ and, since the closure of $C_{c}\left(\mathcal{R}_{\Omega_{\mathcal{N}}}\right)$ with respect to the norm of $\mathcal{A}_{T}$ on the one hand and with respect to reduced norm defined for $\mathcal{R}_{\Omega_{\mathcal{N}}}$ on the other coincide, we have $\left(\mathcal{A}_{T}\right)_{\chi_{\Omega_{\mathcal{N}}}}=C_{r e d}^{*}(\mathcal{R})_{\chi_{\Omega_{\mathcal{N}}}}$. To show that this reduction is full we show that the two sided ideal generated by $\chi_{\Omega_{\mathcal{N}}}$ contains 1 , the unit of the algebra, if $\mathcal{N}$ is regular. By Lemma 1 there is a finite set $\left\{c_{i}\right\}_{i}$ satisfying $\Omega=\dot{\cup}_{i} U_{L\left(c_{i}\right)}$ and $U_{R\left(c_{i}\right)} \subset \Omega_{\mathcal{N}}$. Then $1=\sum_{i} e_{c_{i}} e_{c_{i}^{-1}}=\sum_{i} e_{c_{i}} \chi_{\Omega_{\mathcal{N}}} e_{c_{i}^{-1}}$ showing that 1 is contained in the ideal.

\section{$2.1 \quad K$-theoretical gap labelling}

A local selfadjoint operator $H$ describing the motion of a particle in the tiling $T$ is an operator in the representation $\pi_{T}$ of $\mathcal{A}_{T}$, namely

$$
H \psi\left(T_{x}\right)=\sum_{x^{\prime} \in X(T)} H_{x, x^{\prime}} \psi\left(T_{x^{\prime}}\right) .
$$

Locality refers to the requirement that the matrix element $H_{x, x^{\prime}}$ depends only on a the doubly pointed pattern class of a certain size to which $x, x^{\prime}$ belong, i.e. that $H=\pi_{T}(h)$ for some $h \in C_{c}(\mathcal{R})$. In particular $h$ may be of the form $h=-\Delta+\sum_{i} v_{i} e_{u_{i}}$ where 
$\left\{u_{i}\right\}_{i}$ is a collection of pointed pattern classes and $v_{i} \in \mathbb{R}$ and $\Delta$ is the discrete Laplacian. The latter takes the form $\Delta=\sum_{A \in \mathcal{M}_{\mathbb{I} 2, \neq}} b_{A} e_{A}-\sum_{a \in \mathcal{M}_{\mathbb{I} 1}} b_{a} e_{a}$ where $b_{a} \in \mathbb{R}$ and $b_{A}^{*}=b_{A^{-1}} \in \mathbb{C}$.

The values of the integrated density of states $N_{H}(E)$ of $H$ at energies $E$ lying in a gap of its spectrum serve as labels for the gaps; they are insensitive to certain perturbations of the operator. In Bellissard's $K$-theoretic formulation of the gap labelling [1], 18] these values are recognized as elements of $\operatorname{tr}_{*} K_{0}(\mathcal{A})$ where $\mathcal{A}$ is a $C^{*}$-algebra represented on the above Hilbert space, $H$ an element of its representation, $\operatorname{tr}$ a trace on $\mathcal{A}$, and $\operatorname{tr}_{*}$ the induced state on its $K_{0}$-group $K_{0}(\mathcal{A})$ : The $K_{0}$-group of a unital $C^{*}$-algebra $\mathcal{A}$ is obtained via Grothendieck's construction from the monoid of projection classes of $\mathcal{A} \otimes \mathcal{K}$, i.e. the equivalence classes of projections of $\mathcal{A} \otimes \mathcal{K}$ under $p \sim q$ whenever $\exists u \in \mathcal{A} \otimes \mathcal{K}: p=u u^{*}, q=u^{*} u$ may be added orthogonally, $[p]+[q]=[p \oplus q]$, yielding a monoid $V(\mathcal{A})$, and this monoid may be completed to an abelian group whose elements are classes of pairs under $([p],[q]) \sim\left(\left[p^{\prime}\right],\left[q^{\prime}\right]\right)$ whenever $\exists[r] \in V(A):[p]+\left[q^{\prime}\right]+[r]=$ $[q]+\left[p^{\prime}\right]+[r]$, see [19, 20] for details. Any trace of $\mathcal{A}$ extends to a trace on $\mathcal{A} \otimes \mathcal{K}$ and defines a linear map $\operatorname{tr}_{*}: K_{0}(\mathcal{A}) \rightarrow \mathbb{R}:[p] \mapsto \operatorname{tr}(p)$. The gap labelling in the above formulation requires the equality

$$
N_{H}(E)=\operatorname{tr}\left(\chi_{h \leq E}\right)
$$

$h$ being the element which is represented by $H$ and $\chi_{h \leq E}$ the spectral projection of $h$ to energies smaller or equal to $E$. This equality involves validity of Shubin's formula [18] by which the trace is equated to the operator trace per volume in the corresponding representation. Taking $\mathcal{A}$ to be the algebra associated to the tiling and considering the above representation the $K$-theoretical gap labelling then reads: if $E$ lies in a gap

$$
N_{H}(E) \in \operatorname{tr}_{*} K_{0}\left(\mathcal{A}_{T}\right) \cap[0,1]
$$

provided (43) holds. In other words $\operatorname{tr}_{*} K_{0}\left(\mathcal{A}_{T}\right) \cap[0,1]$ is the set of gap labels predicted by $K$-theory.

One motivation for using $\mathcal{A}_{T}$ is that it is expected not to yield to many values on the r.h.s. so that if the couplings $v_{i}$ are strong and diverse enough all elements of $\operatorname{tr}_{*} K_{0}\left(\mathcal{A}_{T}\right)$ actually occur as labels for open gaps of $H$. In that case, and if $\operatorname{tr}$ is faithful on $\mathcal{A}_{T}$, the density of the values of the integrated density of states on gaps in $[0,1]$ expresses the fact that the continuous part of the spectrum is a Cantor set. In fact, conclusions on the nature of the spectrum may partly be drawn without the need to connect the gap labelling with the values of the integrated density of states, any faithful trace may be used. For instance if $\operatorname{tr}$ is faithful and the set of gap labels $\mathcal{L}_{\operatorname{tr}}(h)=\left\{\operatorname{tr}\left(\chi_{h \leq E}\right) \mid E \notin \sigma(h)\right\}$ dense in $[0,1]$ the spectrum $\sigma(h)$ cannot contain a proper closed interval, for, if $[a, b] \in \sigma(h)$, then by faithfulness $\operatorname{tr}\left(\chi_{h \leq b}\right)>\operatorname{tr}\left(\chi_{h \leq a}\right)-$ here tr has to be extended to measurable functions over $\sigma(h)$ - so that $[0,1] \backslash \mathcal{L}_{\operatorname{tr}}(h)$ would contain the open interval $\left(\operatorname{tr}\left(\chi_{h \leq a}\right), \operatorname{tr}\left(\chi_{h \leq b}\right)\right)$. However, up to now there is no $K$-theoretic formulation of a condition for a Schrödinger operator $h$ under which $\mathcal{L}_{\operatorname{tr}}(h)$ coincides with $\operatorname{tr}_{*} K_{0}\left(\mathcal{A}_{T}\right)$. For a $C^{*}$-algebraic formulation of such a condition for the discrete magnetic Laplacian on $\mathbb{Z}^{2}$ see [21].

Another consequence of the realization of $H$ as an element of a representation of $A_{T}$ is that for minimal $T$ its spectrum has no discrete part, since the spectral projection 
onto the eigenspace of a discrete eigenvalue with finite multiplicity would have to be represented by a compact operator. In fact, for minimal $T$ the algebra $\mathcal{A}_{T}$ is antilimial [22], and hence does not contain compact operators, namely it is neither limial itself its primitive spectrum contains one single point whereas two different representations $\pi_{[\omega]_{o}}$ and $\pi_{\left[\omega^{\prime}\right]_{o}}$ are not unitarily equivalent - nor can it contain a limial ideal.

\section{Traces on $\mathcal{A}_{T}$}

Any normalized trace $\operatorname{tr}$ on $\mathcal{A}_{T}$ restricted to a linear functional $\mu$ on $C(\Omega)$ defines a normalized measure on $\Omega$ also denoted by $\mu$ through $\mu(f)=\operatorname{tr}(f)=\int f d \mu$. This measure is invariant under the groupoid in the sense that $\mu\left(U_{L(c)}\right)=\mu\left(U_{R(c)}\right)$. A direct consequence is that the values of $\mu$ on integer valued continuous functions over $\Omega$ lie in the $\mathbb{Z}$-module generated by the traces of projections of the algebra, and therefore

$$
\mu(C(\Omega, \mathbb{Z})) \subset \operatorname{tr}_{*} K_{0}\left(\mathcal{A}_{T}\right)
$$

The existence of a faithful trace implies that the notion of positive elements of $H(\mathcal{R})=$ $\mathcal{C}(\Omega, \mathbb{Z}) / E_{\mathcal{R}}$ as being those which have a representative which is a positive function turns $H(\mathcal{R})$ into an ordered group. That is the positive elements $H^{+}(\mathcal{R})$ satisfy $H^{+}(\mathcal{R})+$ $H^{+}(\mathcal{R}) \subset H^{+}(\mathcal{R}), H^{+}(\mathcal{R})-H^{+}(\mathcal{R})=H(\mathcal{R})$ and $H^{+}(\mathcal{R}) \cap-H^{+}(\mathcal{R})=\{0\}$. In fact, the existence of a faithful trace rules out that a nonzero element can be both positive and negative. It being zero on $E_{\mathcal{R}}$ it moreover defines a state (if normalized) on $H(\mathcal{R})$. A similar statement holds true for $K_{0}\left(\mathcal{A}_{T}\right)$ : The existence of a normalized faithful trace guarantees that $\mathcal{A}_{T}$ is stably finite and therefore the usual notion of positive elements of $K_{0}\left(\mathcal{A}_{T}\right)$ as those which have a representative which is a projection in $\mathcal{A}_{T} \otimes \mathcal{K}$ turns $K_{0}\left(\mathcal{A}_{T}\right)$ into an ordered group (and the trace induces a state on that group) 19.

Conversely any $\mathcal{R}$-invariant normalized measure $\mu$ on the hull $\Omega$ defines a normalized trace through

$$
\operatorname{tr}(f):=\int_{\Omega} \mathrm{P}(f) d \mu
$$

where $\mathrm{P}: C_{\text {red }}^{*}(\mathcal{R}) \rightarrow C(\Omega)$ is the restriction map. $\mathrm{P}$ is the unique conditional expectation on $C(\Omega)$ and is faithful [10]. Moreover if $T$ is minimal every non trivial invariant measure has to have closed support $\Omega$ so that tr defined by (46) is faithful.

One of the goals of this article is the determination of $\operatorname{tr}_{*} K_{0}\left(\mathcal{A}_{T}\right)$. The question under which circumstances a given trace satisfies Shubin's formula and (43) will not be addressed here, but see [18, 7, 5] for investigations in this directions.

\section{$2.2 \quad K_{0}$-groups for tilings}

The $K_{0}$-group of a $C^{*}$-algebra depends together with its order structure only on its stable isomorphism class. The same holds true for the $K_{1}$-group which may be understood as the $K_{0}$-group of the suspension of $\mathcal{A}$. For this reason we may apply the known results on crossed products to obtain the structure of the $K$-theory for tilings which

reduce to decorations. We are not able to present any results on $K$-groups in case the tiling algebras are not stably isomorphic to crossed products. 
A lot is known about the $K$-groups of crossed products of the form $C(\Omega) \times{ }_{\alpha} \mathbb{Z}^{d}$ in particular for zero dimensional $\Omega$. Recently a relation of these $K$-groups with the group cohomology $H\left(\mathbb{Z}^{d}, C(\Omega, \mathbb{Z})\right)$ of $\mathbb{Z}^{d}$ with coefficients in $C(\Omega, \mathbb{Z})$ was discovered [4]. We will not discuss group cohomology here but what is important for us is that the cohomology group of highest nonvanishing degree (which is $d$ ) coincides with the group of coinvariants of $\Omega \times_{\alpha} \mathbb{Z}^{d}$ and that the $K_{0}$-group decomposes into

$$
K_{0}\left(C(\Omega) \times_{\alpha} \mathbb{Z}^{d}\right) \cong H\left(\Omega \times_{\alpha} \mathbb{Z}^{d}\right) \oplus H^{\prime}
$$

where $H^{\prime}$ is trivial for $d=1$ and equal to $\mathbb{Z}$ in $d=2$. (In general $H^{\prime}$ is a direct sum of cohomology groups of degrees $d-2 n, 0<n \leq \frac{d}{2}$ and $K_{1}\left(C(\Omega) \times_{\alpha} \mathbb{Z}^{d}\right)$ is a direct sum of cohomology groups of degrees $d-1-2 n, 0 \leq n<\frac{d}{2} \llbracket$.) Up to $d=3$ this result was obtained before [23] in an even more explicit form in which in particular becomes clear that the image of the state on $K_{0}\left(C(\Omega) \times_{\alpha} \mathbb{Z}^{d}\right)$ induced by a trace on $C(\Omega) \times_{\alpha} \mathbb{Z}^{d}$ satisfies"

$$
\operatorname{tr}_{*} K_{0}\left(C(\Omega) \times_{\alpha} \mathbb{Z}^{d}\right)=\operatorname{tr}_{*}\left(H\left(\Omega \times_{\alpha} \mathbb{Z}^{d}\right)\right)=\mu(C(\Omega, \mathbb{Z})) .
$$

(48) holds for arbitrary $d$ under the restriction that $\Omega \times_{\alpha} \mathbb{Z}^{d}$ splits into a Cartesian product $\Omega \times{ }_{\alpha} \mathbb{Z}^{d}=\left(\Omega_{1} \times_{\alpha_{1}} \mathbb{Z}\right) \times \ldots \times\left(\Omega_{d} \times_{\alpha_{d}} \mathbb{Z}\right)[5]$.

(47) immediately carries over to tilings which reduce to decorations of $\mathbb{Z}^{d}$ due to Lemma 9

$$
K\left(\mathcal{A}_{T}\right) \cong K\left(C\left(\Omega_{\mathcal{N}}\right) \times_{\alpha} \mathbb{Z}^{d}\right) .
$$

This also concerns the order structure which is however not known for $d>1$.

As for (48) recall that in case the tiling reduces to a decoration $\mathcal{A}_{\mathcal{N}}=C\left(\Omega_{\mathcal{N}}\right) \times{ }_{\alpha} \mathbb{Z}^{d}$ is a subalgebra of $\mathcal{A}_{T}$, i.e. there is an embedding $\imath: \mathcal{A}_{\mathcal{N}} \rightarrow \mathcal{A}_{T}$. This embedding induces an isomorphism $\imath_{*}$ from $K_{0}\left(\mathcal{A}_{\mathcal{N}}\right)$ onto $K_{0}\left(\mathcal{A}_{T}\right)$. In fact, for separable $C^{*}$-algebras, $\mathcal{A}$ being stably isomorphic to $\mathcal{B}$ is equivalent to the existence of a (strong) Morita equivalence $\mathcal{A}$ - $\mathcal{B}$-bimodule which may be viewed as an element of $K K(\mathcal{A}, \mathcal{B})$ and is a special case of a $K K$-equivalence [19, 24, 12]. Any $K K$-equivalence between $\mathcal{A}$ and $\mathcal{B}$ yields an isomophism from $K K(\mathbb{C}, \mathcal{A})$ onto $K K(\mathbb{C}, \mathcal{B})$, namely by multiplying it from the right, the multiplication being the Kasparov product. Translated into $K_{0}$-groups, $K K(\mathbb{C}, \mathcal{A})$ being isomorphic to $K_{0}(\mathcal{A})$, the right multiplication of elements of $K K\left(\mathbb{C}, \mathcal{A}_{\mathcal{N}}\right)$ with the canonical Morita equivalence $\mathcal{A}_{\mathcal{N}}-\mathcal{A}_{T}$-bimodule, which as a linear space is $\mathcal{A}_{\mathcal{N}} \mathcal{A}_{T}$, precisely becomes $\imath_{*}$.

Now $\operatorname{tr} \circ \imath$ is a trace on $\mathcal{A}_{\mathcal{N}}$ which is normalized to $\operatorname{tr}\left(\imath\left(1_{\mathcal{A}_{\mathcal{N}}}\right)\right)=\operatorname{tr}\left(\chi_{\Omega_{\mathcal{N}}}\right)=\mu\left(\Omega_{\mathcal{N}}\right)$. Since the invariant measure on $\Omega_{\mathcal{N}}$ corresponding to $\operatorname{tr} \circ \imath$ is $\left.\mu\right|_{\Omega_{\mathcal{N}}}$ we get

$$
\operatorname{tr}_{*} \circ \imath_{*} K_{0}\left(\mathcal{A}_{\mathcal{N}}\right)=\mu\left(C\left(\Omega_{\mathcal{N}}, \mathbb{Z}\right)\right)
$$

and therefore

$$
\operatorname{tr}_{*} K_{0}\left(\mathcal{A}_{T}\right)=\mu\left(C\left(\Omega_{\mathcal{N}}, \mathbb{Z}\right)\right) \subset \mu(C(\Omega, \mathbb{Z})) .
$$

Together with (45) this extends (48) to tilings which reduce to decorations of $\mathbb{Z}^{d}, d \leq 3$. $K_{0}\left(\mathcal{A}_{\mathcal{N}}\right)$ and $K_{0}\left(\mathcal{A}_{T}\right)$ differ only in their order units (the images of the units of the algebras in $K_{0}$ ). If one identifies them as above the order unit of the former is the $K_{0}$-class of $\chi_{\Omega_{\mathcal{N}}}$.

\footnotetext{
${ }^{1}$ In [23] ergodic measures have been used, but ergodicity not essential for the proof of (48).
} 


\section{Substitution tilings}

We have seen that the integer group of coinvariants furnishes part of the $K$-theory of the algebra of the tiling. In particular in one and two dimensions it yields up to order the $K_{0}$-group. To determine this group we need further structure which is provided by a locally invertible substitution.

A substitution of a tiling may be thought of as a rule according to which the tiles of the tiling are to be replaced by patterns which fit together to yield a new tiling. An algebraic way to formulate this is by means of homomorphisms of the almost-groupoid $\mathcal{M}_{\mathbb{I}}$ incorporating thus their local nature. For the definition recall that $E^{0}(R(c))=$ $\left\{e \in \mathcal{M}_{\mathbb{I}} \mid R(e)=R(c)\right\}$ and that a homomorphism satisfies the growth condition with $t>0$ if $|\operatorname{rad}(\hat{\rho}(u))-t \operatorname{rad}(u)|$ is a bounded function on $\mathcal{M}_{\mathbb{I}}{ }^{0}$. Let $x(c)$ be the first pointed tile of the doubly pointed pattern class $c$.

Definition 13 A substitution of $T$ is a homomorphism of almost-groupoids

$$
\hat{\rho}: \mathcal{M}_{\mathbb{I I}} \rightarrow \mathcal{M}_{\mathbb{I}}
$$

which satisfies the growth condition with $t>1$, and for which the pattern class of $\hat{\rho}(c)$ is composed without overlap (up to boundaries) of the pattern classes of the $\hat{\rho}(x(e))$, $e \in E^{0}(R(c))$, and the first resp. second pointed tile of $\hat{\rho}(c)$ correspond to the pointed tile of $\hat{\rho}(x(c))$ resp. $\hat{\rho}\left(x\left(c^{-1}\right)\right)$.

Since $\hat{\rho}$ satisfies the growth condition it defines a homomorphism of $\mathcal{R}$ into itself. We shall call a tiling resp. its class invariant under a substitution if $[\rho]_{o}(T)=T$. We call it a substitution tiling if it allows for a substitution such that $[\rho]_{o}(T)$ is locally isomorphic to $T$.

Like any homomorphism $\hat{\rho}$ is determined by its action on the generators $\mathcal{M}_{\mathbb{I} 2, \neq}$. But by the above definition this means that $\hat{\rho}$ may be given, first, by its image on the pointed pattern classes of tiles, $\mathcal{M}_{\mathbb{I} 1}$, and second, by the relative position of these images.

A substitution can be iterated and it is called primitive if for some $n$ and all $a \in \mathcal{M}_{\mathbb{I I} 1}$ the pattern class $\rho^{n}(a)$ contains at least one tile of each tile class.

If a tiling allows for a substitution which has a right inverse, and this is equivalent with locally invertible as we have seen, then the substitution can be used to compute the coinvariants associated to the tiling class. We shall carry this out below but first give a brief discussion on geometric realizations of substitutions.

\subsection{Deflation}

Geometrically we view a pointed tiling as a representative of $T$ in the Euclidian space $\mathbb{R}^{d}$ with fixed origin 0 . To be precise we choose a point for each tile class (its puncture). Then $T_{x}$ shall correspond to the representative of $T$ for which the puncture of the pointed tile coincides with 0 thereby getting a bijective correspondance between pointed tilings and representatives in $\mathbb{R}^{d}$ having the puncture of one of its tiles at 0 . Substitutions appear then as deflations followed by rescaling. Let $t^{-1} T$ denote $T$ rescaled by $t^{-1}$ and $\mathcal{M}(T)$ the (unpointed) pattern classes of $T$. A deflation is given by: 
1 a pattern class $\rho_{t}(a) \in \mathcal{M}\left(t^{-1} T\right)$ for each tile class $a, t>0$.

2 a relative position between $a$ and $\rho_{t}(a)$ for each tile class $a$.

Let $\mathcal{T}$ be a representative of $T$ in $\mathbb{R}^{d}$. In the process of applying the deflation any tile of class $a$ in $\mathcal{T}$ is to be replaced by a pattern of class $\rho_{t}(a)$ (its replacement) at the relative position given above. To be more precise choose a tile in $\rho_{t}(a)$. Its puncture shall now indicate the position of a pattern of class $\rho_{t}(a)$ in $\mathbb{R}^{d}$. Then the relative position i.e. the difference between the position of the tile and its replacement shall be $x_{a} \in \mathbb{R}^{d}$. Thus if a tile of $\mathcal{T}$ of class $a$ is at $x \in \mathbb{R}$ then a pattern of class $\rho_{t}(a)$ is in $\rho_{t}(\mathcal{T})$ at $x-x_{a}$. The object $\rho_{t}(\mathcal{T})$ is a composition of patterns of the classes $\rho_{t}(a)$, and it is required that this yields a tiling which is locally isomorphic to $t^{-1} T$. In particular no overlap and no gaps are allowed.

Such a deflation defines a substitution in the algebraic sense (as a homomorphism of $\left.\mathcal{M}_{\mathrm{II}}\right)$. By the above choice of a tile in $\rho_{t}(a)$ the latter may now be understood as a pointed pattern class of $t^{-1} T$. We let $\hat{\rho}(a)$ be $t \rho_{t}(a)$ which is $\rho_{t}(a)$ scaled by $t$, the pattern class of $\hat{\rho}\left(M_{x_{1} x_{2}}\right)$ be $t \rho_{t}(M)$, and the first resp. second pointed tile be the one corresponding to the pointed tile of $\hat{\rho}\left(x\left(M_{x_{1} x_{2}}\right)\right)$ resp. $\hat{\rho}\left(x\left(M_{x_{2} x_{1}}\right)\right)$.

A deflation is locally invertible if the determination of whether or not a pattern of $\rho_{t}(\mathcal{T})$ is a replacement of a tile of $\mathcal{T}$ may be uniquely carried out by inspection of the larger patterns around that pattern up to a given finite size. In other words, for each $a$ there is a finite set of pointed pattern classes $\Phi_{t}(a) \subset \mathcal{M}_{\mathbb{I}}\left(t^{-1} T\right)^{0}$ with $\forall v \in \Phi_{t}(a): \rho_{t}(a) \preceq v$ such that whenever one of them occurrs in $\rho_{t}(\mathcal{T})$ at $x \in \mathbb{R}$ then a tile of class $a$ occurrs in $\mathcal{T}$ at $x+x_{a}$. This condition allows one to locally obtain a preimage of any representative of any $t^{-1} T^{\prime}, T^{\prime} \in[\Omega]_{o}$. But this furnishes also a right inverse of $\rho$ on $\Omega$ as follows: Let $\mathcal{T}$ be the representative corresponding to $\omega \in \operatorname{im} \rho$. Then a preimage of $\omega$ under $\rho$ is given by the pointed tiling corresponding to $\rho_{t}^{-1}\left(t^{-1} \mathcal{T}\right)-x_{a}$ where $t^{-1} \mathcal{T}$ is rescaled in such a way that the puncture remains on 0 and $a$ is determined through the unique replacement $\rho_{t}(a)$ whose puncture is on 0 . Clearly the right inverse of $\rho$ on $\Omega$ extends to $\mathcal{S}$. And since non periodicity is forced by the existence of a locally invertible deflation [11, 5] the corresponding substitution $\hat{\rho}$ is locally invertible in the sense of section 1.4.

\subsection{Path spaces over graphs and their dimension groups}

This section is meant to fix the notation thereby giving an overview on the structures that will be needed.

A graph $\Sigma$ is a set of vertices $\Sigma^{(0)}$ and a set of edges $\Sigma^{(1)}$ with two maps $s, r: \Sigma^{(1)} \rightarrow$ $\Sigma^{(0)}$, the source and the range map. Its connectivity matrix is the $\left|\Sigma^{(0)}\right| \times\left|\Sigma^{(0)}\right|$ matrix with coefficients

$$
\sigma_{x y}:=\text { number of edges which have source } y \text { and range } x \text {. }
$$

A path $\xi=\xi_{1} \ldots \xi_{n}$ of length $n$ over $\Sigma$ is a sequence of $n$ edges such that $r\left(\xi_{k}\right)=s\left(\xi_{k+1}\right)$. We denote its lentgh by $|\xi|$. One sets $r(\xi)=r\left(\xi_{n}\right)$ and $s(\xi)=s\left(\xi_{1}\right)$. Two paths $\xi, \xi^{\prime}$ with $r(\xi)=s\left(\xi^{\prime}\right)$ may be concatenated to yield longer path $\xi \circ \xi^{\prime}=\xi_{1} \ldots \xi_{|\xi|} \xi_{1}^{\prime} \ldots \xi_{\left|\xi^{\prime}\right|}^{\prime}$. 
The set of all (half) infinite paths over the graph yields a space which carries a compact metric topology, it is called the path space $\mathcal{P}_{\Sigma}$ of the graph. Its topology is generated by sets

$$
U_{\xi}=\{\xi \circ \gamma \mid s(\gamma)=r(\xi)\} .
$$

These sets are closed as well so that $\mathcal{P}_{\Sigma}$ is a zero dimensional. Two (infinite) paths $\gamma, \gamma^{\prime}$ are called confinal if for some $n, \forall i \geq n: \gamma_{i}=\gamma_{i}^{\prime}$. Cofinality is an equivalence relation and the subspace of $\mathcal{P}_{\Sigma} \times \mathcal{P}_{\Sigma}$ of all cofinal pairs may be given a topology which is generated by

$$
\mathcal{U}_{\xi \xi^{\prime}}=\left\{\left(\xi \circ \gamma, \xi^{\prime} \circ \gamma\right) \mid s(\gamma)=r(\xi)\right\}
$$

where it is required that $|\xi|=\left|\xi^{\prime}\right|$ and $r(\xi)=r\left(\xi^{\prime}\right)$. With this topology the groupoid defined by the equivalence relation becomes an $r$-discrete principal groupoid $\mathcal{R}_{\Sigma}$. Its groupoid- $C^{*}$-algebra $\mathcal{A}_{\Sigma}$ is finitely approximated, i.e. an $A F$-algebra. The integer group of coinvariants of $H\left(\mathcal{R}_{\Sigma}\right)$ of this groupoid is $C\left(\mathcal{P}_{\Sigma}, \mathbb{Z}\right) / E_{\Sigma}$ where $E_{\Sigma}$ is generated by elements of the form $\eta\left(\mathcal{U}_{\xi \xi^{\prime}}\right)=\chi_{U_{\xi}}-\chi_{U_{\xi^{\prime}}} \cdot C\left(\mathcal{P}_{\Sigma}, \mathbb{Z}\right) / E_{\Sigma}$ carries an order structure given by the notion of positive functions, e.g. $[f]_{\Sigma}>0$ whenever it has a representative $f>0$. With that order structure and the constant function 1 representing the order unit $H\left(\mathcal{R}_{\Sigma}\right)$ is also the dimension group or scaled ordered $K_{0}$-group of the $A F$-algebra $\mathcal{A}_{\Sigma}$ [25]. We will not discuss the $C^{*}$-algebraic and $K$-theoretic details here but only concentrate on the computation of the dimension group which will be used lateron to determine the coinvariants of $\mathcal{R}$.

The main point is that $H\left(\mathcal{R}_{\Sigma}\right)$ can be obtained by taking the algebraic limit of the directed system $\left(G_{n}, \sigma\right)$ where $G_{n}=\mathbb{Z}^{\left|\Sigma^{(0)}\right|}$ and $\sigma: G_{n} \rightarrow G_{n+1}$ is the homomorphism given by the connectivity matrix once the vertices have been identified with the standard base. The algebraic limit of the above system is a universal object which is a group $G$ together with homomorphisms $j_{n}: G_{n} \rightarrow G$ such that $j_{n+1} \circ \sigma=j_{n}$ [26]. It is up to isomorphism determined by the property that, if there is any other group $G^{\prime}$ and $j_{n}^{\prime}: G_{n} \rightarrow G^{\prime}$ such that $j_{n+1}^{\prime} \circ \sigma=j_{n}^{\prime}$ then there is a unique homomorphism $j: G \rightarrow G^{\prime}$ such that $j_{n}^{\prime}=j \circ j_{n}$. Moreover, $G$ inherits an order structure and the order unit from the standard order structures on the $G_{n}$. And $H\left(\mathcal{R}_{\Sigma}\right)$ coincides with $G$ as ordered group with order unit.

There are several "standard" realizations for this limit [26, 20]. We will use neither of them here but instead one which is less general but more suitable for our means, c.f. [9]. In this realization the group is isomorphic to the quotient

$$
H\left(\mathcal{R}_{\Sigma}\right) \cong\left\{x \in \mathbb{R}^{k} \mid \exists n \geq 0: \sigma^{n}(x) \in \mathbb{Z}^{k}\right\} /\left\{x \in \mathbb{R}^{k} \mid \exists n \geq 0: \sigma^{n}(x)=0\right\}
$$

with $k=\left|\Sigma^{(0)}\right|$. The maps $j_{n}: G_{n}=\mathbb{Z}^{k} \rightarrow H\left(\mathcal{R}_{\Sigma}\right)$ are given by $j_{n}=\pi \circ \sigma^{-n}$ where $\sigma^{-1}$ is taking the preimage. If $G^{\prime}$ and $j_{n}^{\prime}: G_{n} \rightarrow G^{\prime}$ with $j_{n+1}^{\prime} \circ \sigma=j_{n}^{\prime}$ is any other realization then $j(\pi(x))=j_{n}^{\prime}\left(\sigma^{n}(x)\right)$ for $\sigma^{n}(x) \in \mathbb{Z}^{k}$ yields the unique homomorphism $j: H\left(\mathcal{R}_{\Sigma}\right) \rightarrow G^{\prime}$ satisfying $j_{n}^{\prime}=j \circ j_{n}$. To express the order structure in this realization we restrict for simplicity to the case where $\sigma$ is primitive so that it has a Perron-Frobenius-eigenvalue $\tau$ with left-Perron-Frobenius-eigenvector $\nu$. If $\pi$ denotes the canonical projection of the above quotient, the positive cone is

$$
H^{+}\left(\mathcal{R}_{\Sigma}\right) \cong\left\{\pi(x) \in H\left(\mathcal{R}_{\Sigma}\right) \mid \sum_{i} \nu_{i} x_{i}>0\right\} \cup\{0\}
$$


and the order unit $\pi(w) \in H\left(\mathcal{R}_{\Sigma}\right)$ with $w_{i}=1$.

Since $\sigma$ is primitive, there is a unique normalized measure $\mu$ on $\mathcal{P}_{\Sigma}$ which is invariant under the groupoid $\mathcal{R}_{\Sigma}$, i.e. satisfies $\mu\left(U_{\xi}\right)=\mu\left(U_{\xi^{\prime}}\right)$ in case $|\xi|=\left|\xi^{\prime}\right|$ and $r(\xi)=r\left(\xi^{\prime}\right)$ [25]. If the left Perron-Frobenius-eigenvector of $\sigma$ normalized to $\sum_{i} \nu_{i}=1$ then $\mu\left(U_{\xi}\right)=$ $\tau^{-|\xi|} \nu_{r(\xi)}$ and its range on $C\left(\mathcal{P}_{\Sigma}, \mathbb{Z}\right)$ is given by

$$
\mu\left(C\left(\mathcal{P}_{\Sigma}, \mathbb{Z}\right)\right)=\left\{\tau^{-n} \sum_{i} \nu_{i} n_{i} \mid n \geq 0, n_{i} \in \mathbb{Z}\right\}
$$

In particular $\mu$ is well defined on $H\left(\mathcal{R}_{\Sigma}\right)$ and the order may be expressed as

$$
H^{+}\left(\mathcal{R}_{\Sigma}\right)=\left\{x \in H\left(\mathcal{R}_{\Sigma}\right) \mid \mu(x)>0\right\} \cup\{0\}
$$

Elements which are neither positive nor negative are called infinitesimal.

\subsection{Path spaces determined by substitutions}

Any substitution, invertible or not, defines an obvious graph which however does not even for all locally invertible substitutions contain enough information of the tiling. In analogy to the one dimensional case we call it with [8] the improper graph. It has the substitution matrix as connectivity matrix. But other graphs may also be attached to the substitution. These are related to the improper graph and coincide with it in the border forcing case. They allow for a coding of the tilings which yields a homeomorphism between their path space and the hull. This will be used to solve the $K$-theoretical gap labelling for a certain class of substitution tilings.

Let for $a \in \mathcal{M}_{\mathrm{II} 1}$

$$
E^{n}(a)=\left\{e \in \mathcal{M}_{\mathbb{I}} \mid R(e)=\hat{\rho}^{n}(a)\right\} .
$$

The improper graph $\Sigma$ is the graph which has vertices and edges resp.

$$
\begin{aligned}
& \Sigma^{(0)}=\mathcal{M}_{\mathbb{I} 1} \\
& \Sigma^{(1)}=\bigcup_{a \in \mathcal{M}_{\mathbb{I}_{1}}}\left\{(e, a) \mid e \in E^{1}(a)\right\}
\end{aligned}
$$

and range resp. source maps $r, s: \Sigma^{(1)} \rightarrow \Sigma^{(0)}$ given by

$$
\begin{aligned}
& r(e, a)=a \\
& s(e, a)=x(e) .
\end{aligned}
$$

Thus the edges of the graph which have range $a$ are in bijective correspondence to the tiles in $\hat{\rho}(a)$. $\Sigma$ has as connectivity matrix

$$
\sigma_{a_{1} a_{2}}=\left|\left\{e \in E^{1}\left(a_{1}\right) \mid x(e)=a_{2}\right\}\right|,
$$

i.e. $\sigma_{a_{1} a_{2}}$ equals the number of $a_{2}$ 's in $\hat{\rho}\left(a_{1}\right)$. This matrix (or sometimes its transpose) is also called the substitution matrix of $\hat{\rho}$. A path of finite length like $\left(\left(e_{1}, a_{1}\right), \ldots,\left(e_{n}, a_{n}\right)\right)$ depends due to the particular form of the range map only on $\left(e_{1}, \ldots, e_{n}\right)$ and $a_{n}$. It shall be written shorter $\left(e_{1}, \ldots, e_{n} ; a_{n}\right)$. 
The improper graph does not contain enough information of the tiling to yield the right integer group of coinvariants. To improve this we have to incorporate the neighborhood of substitutes. To a given doubly pointed pattern class $c$ let $\mathcal{F}(c)$ be the set of all possible neighborhoods of $c$, i.e. the set of pattern classes occuring in $T$ which are composed of $c$ together with all the tiles the boundaries of which have a non empty intersection of with $c$. The pointed tiles are those of $c$.

An integer $v \geq 0$ will parametrize a set of graphs $\Lambda_{v}$. (This generality is needed lateron for applications.) Define

$$
\mathcal{B}_{1}^{v}:=\left\{(a, f) \mid a \in \mathcal{M}_{\mathbb{I} 1}, f \in \mathcal{F}\left(\hat{\rho}^{v}(a)\right), \rho^{v}\left(U_{a}\right) \cap U_{f} \neq \emptyset\right\} .
$$

The extra condition insures that only those neighborhoods of $\hat{\rho}^{v}(a)$ are taken into account which are neighborhoods of the $v$-fold substitute of $a$. Let $\Lambda_{v}$ be the graph with vertices and edges

$$
\begin{aligned}
& \Lambda_{v}^{(0)}=\mathcal{B}_{1}^{v} \\
& \Lambda_{v}^{(1)}=\left\{(e, a, f) \mid(a, f) \in \mathcal{B}_{1}^{v}, e \in E^{1}(a)\right\}
\end{aligned}
$$

and range and source map given by

$$
\begin{aligned}
r(e, a, f) & =(a, f) \\
s(e, a, f) & =\left(x(e), f^{\prime}\right) \text { with } f^{\prime} \preceq L\left(\hat{\rho}^{v}(e) \hat{\rho}(f)\right) .
\end{aligned}
$$

(The $f^{\prime} \in \mathcal{F}\left(\hat{\rho}^{v}(a)\right)$ for which $f^{\prime} \preceq L\left(\hat{\rho}^{v}(e) \hat{\rho}(f)\right)$ is unique.) The connectivity matrix $\lambda_{v}$ of $\Lambda_{v}$ has coefficients

$$
\lambda_{v\left(a_{1}, f_{1}\right)\left(a_{2}, f_{2}\right)}=\left|\left\{e \in E^{1}\left(a_{1}\right) \mid x(e)=a_{2}, f_{2} \preceq L\left(\hat{\rho}^{v}(e) \hat{\rho}\left(f_{1}\right)\right)\right\}\right| .
$$

Like for $\Sigma$ a path $\left(\left(e_{1}, a_{1}, f_{1}\right), \ldots,\left(e_{n}, a_{n}, f_{n}\right)\right)$ depends only on $\left(e_{1}, \ldots, e_{n}\right)$ and $\left(a_{n}, f_{n}\right)$ and may be abbreviated as $\left(e_{1}, \ldots, e_{n} ; a_{n}, f_{n}\right)$. Note that such a path satisfies $R\left(e_{k}\right)=$ $\hat{\rho}\left(x\left(e_{k+1}\right)\right)$ and $R\left(e_{n}\right)=\hat{\rho}\left(a_{n}\right)$. Therefore the map $\delta: \mathcal{P}_{\Lambda_{v}}^{n}(a, f) \rightarrow E^{n}(a)$ given by

$$
\delta\left(e_{1}, \ldots, e_{n} ; a, f\right):=e_{1} \hat{\rho}\left(e_{2}\right) \ldots \hat{\rho}^{n-1}\left(e_{n}\right)
$$

is a bijection for fixed $(a, f)$. Furthermore let $\beta_{v}: \mathcal{B}_{1}^{v} \rightarrow \mathcal{M}_{\mathbb{I I} 1}$ :

$$
\beta_{v}(a, f)=a
$$

Extending $\beta_{v}$ first to edges via $\beta_{v}(e, a, f):=(e, a)$ and then to paths on obtains a map $\beta_{v}: \mathcal{P}_{\Lambda_{v}}^{n} \rightarrow \mathcal{P}_{\Sigma}^{n}:$

$$
\beta_{v}\left(e_{1}, \ldots, e_{n} ; a_{n}, f_{n}\right)=\left(e_{1}, \ldots, e_{n} ; a_{n}\right)
$$

which is not only surjective but also injective on $\mathcal{P}_{\Lambda_{v}}^{n}(a, f)$ for fixed $f$. In particular $\beta_{v}$ extends to a continuous surjective map from $\mathcal{P}_{\Lambda_{v}}$ onto $\mathcal{P}_{\Sigma}$.

Lemma 10 Let $T$ be a substitution tiling with locally invertible substitution $\hat{\rho}$. For each $\omega \in \Omega$ there is a unique $(e, a) \in \Sigma^{(1)}$ such that $\omega \cdot e \in \operatorname{im} \rho$. 
Proof: By definition of a substitution does for any $c \in \mathcal{M}_{\mathrm{II}}$ hold the alternative: either $c=c^{-1}$ or $\forall e_{i} \in E^{1}\left(x_{i}(c)\right): e_{1} \rho(c) e_{2}^{-1} \neq\left(e_{1} \rho(c) e_{2}^{-1}\right)^{-1}$ where $x_{1}(c)=x(c)$ and $x_{2}(c)=x\left(c^{-1}\right)$. Recall from the proof of Theorem 11 that $\rho(\Omega)=\Omega_{\mathcal{N}}$ where $\mathcal{N}=\Phi\left(\mathcal{M}_{\mathbb{I}}\right)$. Since for all $d \in \Phi(c), \hat{\rho}(c)$ and $d$ have a common greater element the above alternative carries over for any $d \in \mathcal{N}$ in the form: either $d=d^{-1}$ or $\forall e_{i} \in E^{1}\left(x_{i}(d)\right): e_{1} d e_{2}^{-1} \neq\left(e_{1} d e_{2}^{-1}\right)^{-1}$. Since $[\rho]_{o}(T)$ is locally isomorphic to $T, \mathcal{N}$ has to be regular and there is a $c \in \mathcal{M}_{\mathbb{I}}$ such that $\omega \cdot c \in \Omega_{\mathcal{N}}$. And as $\mathcal{N}$ is approximating and generating ( $T$ has to be non periodic) $c$ can be written as $c=e d$ where $d \in \mathcal{N}$ and $e \in E^{1}(x(d))$. We claim that $e$ is unique, for if not then $L(c)=e d d^{\prime-1} e^{\prime-1}$ and since this is a unit $d d^{\prime-1}$ must be equal to its inverse by the above alternative. Hence $e=e^{\prime}$. Finally, if $\omega \cdot e d \in \operatorname{im} \rho$ then also $\omega \cdot e \in \operatorname{im} \rho$. The condition $a \preceq \rho^{-1}(\omega \cdot e)$ determines uniquely the $a$ for which $e \in E^{1}(a)$.

Let $\kappa_{v}: \Omega \rightarrow \Lambda_{v}^{(1)}: \omega \mapsto(e, a, f)$ where $(e, a)$ is determined by the above lemma and $f \preceq \rho^{v-1}(\omega \cdot e)$. Furthermore, the first component of $(e, a)$ shall be used to define an extension of $\rho^{-1}$ to all of $\Omega$ through

$$
\psi(\omega):=\rho^{-1}(\omega \cdot e) .
$$

Lemma $11 s\left(\kappa_{v}\left(\psi^{n}(\omega)\right)\right)=r\left(\kappa_{v}\left(\psi^{n-1}(\omega)\right)\right.$.

Proof: Let $\kappa_{v}\left(\psi^{n}(\omega)\right)=\left(e_{2}, a_{2}, f_{2}\right)$ and $\kappa_{v}\left(\psi^{n-1}(\omega)\right)=\left(e_{1}, a_{1}, f_{1}\right)$. We have to show that, first, $x\left(e_{2}\right)=a_{1}$, and second, that the $f^{\prime} \in \mathcal{F}\left(\hat{\rho}^{v}\left(a_{2}\right)\right)$ which satisfies $f^{\prime} \preceq$ $L\left(\hat{\rho}^{v}\left(e_{2}\right) \hat{\rho}\left(f_{2}\right)\right)$ equals to $f_{1}$. The first follows from the definitions: $a_{1} \preceq \rho^{-1}\left(\psi^{n-1}(\omega)\right.$. $\left.e_{1}\right)=\psi^{n}(\omega)$ and $L\left(e_{2}\right) \preceq \psi^{n}(\omega)$. As for the second, $f_{2} \preceq \rho^{v-1}\left(\psi^{n}(\omega) \cdot e_{2}\right)$ implies that $\rho\left(L\left(\hat{\rho}^{v-1}\left(e_{2}\right) f_{2}\right)\right) \preceq \rho^{v}\left(\psi^{n}(\omega)\right)=\rho^{v-1}\left(\psi^{n-1}(\omega) \cdot e_{1}\right)$. Hence $f^{\prime} \preceq \rho^{v-1}\left(\psi^{n-1}(\omega) \cdot e_{1}\right)$ which is the relation determining $f_{1}$.

As a consequence we may define a coding which is a map $Q_{v}: \Omega \rightarrow \mathcal{P}_{\Lambda_{v}}$ where the $n$th edge of $Q_{v}(\omega)$ is

$$
Q_{v}(\omega)_{n}:=\kappa_{v}\left(\psi^{n-1}(\omega)\right)
$$

Theorem 3 Under the conditions of Lemma 10 is $Q_{v}: \Omega \rightarrow \mathcal{P}_{\Lambda_{v}}$ a homeomorphism.

Proof: Local invertibility implies that, for $n \geq v$

$$
\mathcal{V}_{v}^{n}\left(a, f, e_{1}, e_{2}\right):=\left\{\left[\omega \cdot e_{1}^{-1}, e_{1} \hat{\rho}^{n-v}(f) e_{2}^{-1}\right] \mid \omega \in \rho^{n}\left(U_{a}\right) \cap \rho^{n-v}\left(U_{f}\right)\right\}
$$

is compact and open for all $(a, f) \in \mathcal{B}_{1}^{v}$ and $e_{i} \in E^{n}(a)$. (In fact, these are $\mathcal{R}$-sets.) Let $\xi$ be a path of length $n$ with $r(\xi)=(a, f) . Q_{v}(\omega) \in U_{\xi}$ if and only if $L(\delta(\xi)) \preceq \omega$, $a \in \psi^{n}(\omega)$, and $f \preceq \rho^{v}\left(\psi^{n}(\omega)\right)$. Since $\omega \cdot \delta(\xi) \in \operatorname{im} \rho^{n}$ and $\psi^{n}(\omega)=\rho^{-n}(\omega \cdot \delta(\xi))$ this means

$$
Q_{v}^{-1}\left(U_{\xi}\right)=\mathcal{V}_{v}^{|\xi|}(r(\xi), \delta(\xi), \delta(\xi))
$$


Hence $Q_{v}$ is continuous. Since $Q_{v}^{-1}\left(U_{\xi}\right)$ is not empty, any path over $\Lambda_{v}$ is an accumulation point of $\operatorname{im} Q_{v}$. As the latter is closed $Q_{v}$ is surjective. Finally, to prove injectivity, let $r\left(Q_{v}(\omega)_{k}\right)=\left(a_{k}, f_{k}\right)$ and $\tilde{e}_{n}:=\delta\left(Q_{v}(\omega)_{1} \ldots Q_{v}(\omega)_{n}\right)$. Then $\hat{\rho}^{n-v}(f) \preceq \omega \cdot \tilde{e}_{n}$ and

$$
u_{n}(\omega):=L\left(\tilde{e}_{n} \hat{\rho}^{n-v}\left(f_{n}\right)\right) \preceq \omega .
$$

Since $L\left(\tilde{e}_{n} \hat{\rho}^{n}\left(a_{n}\right)\right) \preceq L\left(\tilde{e}_{n} \hat{\rho}^{n-v}\left(f_{n}\right)\right)$ the radius of $u_{n}(\omega)$ diverges exponentionally with $n$. Hence $u_{n}(\omega)$ is an approximation which approximates $\omega$ and is uniquely determined by $Q_{v}(\omega)$. Thus $Q_{v}$ is injective.

Theorem 3 implies that $Q_{v}^{*}: C\left(\mathcal{P}_{\Lambda_{v}}, \mathbb{Z}\right) \rightarrow C(\Omega, \mathbb{Z}): Q_{v}^{*}(f)=f \circ Q_{v}^{-1}$ is an isomorphism of groups. We may extend $Q_{v}$ to a map $Q_{v}: \mathcal{S} \rightarrow \mathcal{S}_{\Lambda_{v}}$ by restricting $Q_{v} \times Q_{v}$ to $\mathcal{S}$ and since $T$ is not periodic we may view it as a map $Q_{v}: \mathcal{R} \rightarrow \mathcal{R}_{\Lambda_{v}}$. Then alike (78)

$$
Q_{v}^{-1}\left(\mathcal{U}_{\xi \xi^{\prime}}\right)=\mathcal{V}_{v}^{|\xi|}\left(r(\xi), e_{\xi}, e_{\xi^{\prime}}\right)
$$

showing that this map is continuous and surjective, but it is not injective. Since $\eta \circ Q_{v}^{-1}=Q_{v}^{*} \circ \eta$ the image under $Q_{v}^{*}$ of the subgroup $E_{\Lambda_{v}}$ is contained in $E_{\mathcal{R}}$. Hence there is an induced surjective homomorphism $\left[Q_{v}^{*}\right]: H\left(\mathcal{R}_{\Lambda_{v}}\right) \rightarrow H(\mathcal{R})$. Its kernel is $E_{\mathcal{R}} / Q_{v}^{*}\left(E_{\Lambda_{v}}\right)$. In particular, since $\lambda_{v}$ is primitive, we get a corrolary from Theorem 3 :

Corrolary 1 Let $T$ be a substitution tiling with primitive locally invertible substitution $\hat{\rho}$ which reduces to a decoration of $\mathbb{Z}^{d}, d \leq 3$, and tr be a trace on $A_{T}$. Then

$$
\operatorname{tr}_{*}\left(K_{0}\left(\mathcal{A}_{T}\right)\right)=\left\{\tau^{-n} \sum_{i} \nu_{i} n_{i} \mid n>0, n_{i} \in \mathbb{Z}\right\}
$$

where $\tau$ is the Perron-Frobenius-eigenvalue and $\nu$ the left Perron-Frobenius-eigenvector of $\lambda_{v}$ normalized to $\sum_{i} \nu_{i}=1$.

Proof: If $\mu$ is the measure on $\Omega$ obtained by restricting the trace then $\mu \circ Q_{v}^{-1}$ is a measure on $\mathcal{P}_{\Lambda_{v}}$ which is invariant under $\mathcal{R}_{\Lambda_{v}}$. Using (48) one obtains $\operatorname{tr}_{*}\left(K_{0}\left(\mathcal{A}_{T}\right)\right)=$ $\mu\left(Q_{v}^{-1}\left(C\left(\mathcal{P}_{\Lambda_{v}}, \mathbb{Z}\right)\right)\right)$ and with $(58)$ the statement.

This solves the $K$-theoretical version of the gap labelling for substitution tilings with primitive locally invertible substitution.

Remark: Let us only remark here that one obtains not only the above embedding of groups by $Q_{v}^{*}$ but in fact a unital embedding $i: \mathcal{A}_{\Lambda_{v}} \rightarrow \mathcal{A}_{T}$ of the $A F$-algebra defined by $\Lambda_{v}$ into the algebra associated to the tiling similar to embedding of $\mathcal{A}_{\Sigma}$ in $\mathcal{A}_{T}$ described in [5]. In fact, a glance on (79) tells us that the characteristic functions on $\mathcal{V}_{v}^{|\xi|}\left(r(\xi), e_{\xi}, e_{\xi^{\prime}}\right)$ generate $\mathcal{A}_{\Lambda_{v}}$ as a subalgebra of $\mathcal{A}_{T}$. The embedding induces an order homomorphism $i_{*}$ of $K$-groups. Then $K_{0}\left(\mathcal{A}_{\Lambda_{v}}\right)=H\left(\mathcal{R}_{\Lambda_{v}}\right)$ as ordered group with order unit, and, under the hypothesis (47), $i_{*}: K_{0}\left(\mathcal{A}_{\Lambda_{v}}\right) \rightarrow H(\mathcal{R})$ coincides with $\left[Q_{v}^{*}\right]$. However, in case $i_{*}$ is not surjective, i.e. the dimension of the tiling is bigger than one, our analysis will not determine the order structure on $K_{0}\left(\mathcal{A}_{T}\right)$ but only a subcone of the positive cone $K_{0}^{+}\left(\mathcal{A}_{T}\right)$. 


\subsection{The integer group of coinvariants for substitution tilings}

Recall that the integer group of coinvariants is given by $H(\mathcal{R})=H\left(\mathcal{R}_{\Lambda_{v}}\right) / \operatorname{ker}\left[Q_{v}^{*}\right]$. To compute $\operatorname{ker}\left[Q_{v}^{*}\right]=E_{\mathcal{R}} / Q_{v}^{*}\left(E_{\Lambda_{v}}\right)$ we need to control pattern classes consisting of two tiles. In analogy to (66, ,77) define' for $n \geq v$

$$
\begin{gathered}
\mathcal{B}_{2 \neq}^{v}:=\left\{(A, F) \mid A \in \mathcal{M}_{\mathbb{I I} 2, \neq}, F \in \mathcal{F}\left(\hat{\rho}^{v}(A)\right), \rho^{v}\left(U_{A}\right) \cap U_{F} \neq \emptyset\right\} . \\
\mathcal{V}_{v}^{n}\left(A, F, e_{1}, e_{2}\right):=\left\{\left[\omega \cdot e_{1}^{-1}, e_{1} \hat{\rho}^{n-v}(F) e_{2}^{-1}\right] \mid\left[\omega, \hat{\rho}^{n-v}(F)\right] \in \rho^{n}\left(U_{A}\right) \cap \rho^{n-v}\left(U_{F}\right)\right\}
\end{gathered}
$$

where $e_{i} \in E^{n}\left(x_{i}(A)\right), x_{1}(c)=x(c)$ and $x_{2}(c)=x\left(c^{-1}\right)$. If $e_{i}$ are units we write $\mathcal{V}_{v}^{n}(A, F)$ and resp. $\mathcal{V}_{v}^{n}(a, f)$ for the above. It should be clear that for fixed $e_{1}, e_{2}$ the sets $\mathcal{V}_{v}^{n}\left(x, y, e_{1}, e_{2}\right)$ are for different $(x, y) \in \mathcal{B}_{1}^{v} \cup \mathcal{B}_{2 \neq}^{v}$ pairwise disjoint. [ $\left.\cdot\right]_{\Lambda_{v}}$ denotes equivalence classes with respect to $Q_{v}^{*}\left(E_{\Lambda_{v}}\right)$.

Lemma 12 Let $T$ be a substitution tiling with locally invertible substitution and $\Lambda_{v}$ and $Q_{v}$ as above. Then $E_{\mathcal{R}} / Q_{v}^{*}\left(E_{\Lambda_{v}}\right)$ is generated by elements of the form $\left[\eta\left(\mathcal{V}_{v}^{n}(A, F)\right)\right]_{\Lambda_{v}}$ for $(A, F) \in \mathcal{B}_{2 \neq}^{v}$.

Proof: Since $\mathcal{M}_{\mathbb{I} 2, \neq}$ generates $\mathcal{M}_{\mathbb{I}} \backslash \mathcal{M}_{\mathbb{I} 1}$ a generating set for $E_{\mathcal{R}}$ is provided by the set of elements of the form $\eta\left(\chi_{c}\right)$ where $c=u A, u \in \mathcal{M}_{\mathbb{I}}{ }^{0}, u \vdash A \in \mathcal{M}_{\mathbb{I} 2, \neq}$. For such a $c$ let

$$
\begin{aligned}
I_{1}^{n}(c) & :=\left\{\left(a, f, e_{1}, e_{2}\right)\left|(a, f) \in \mathcal{B}_{1}^{v}, e_{i} \in E^{n}(a)\right| \exists c^{\prime}: c, e_{1} \hat{\rho}^{n-v}(f) e_{2}^{-1} \preceq c^{\prime}\right\} \\
I_{2 \neq}^{n}(c) & :=\left\{\left(A, F, e_{1}, e_{2}\right)\left|(A, F) \in \mathcal{B}_{2 \neq}^{v}, e_{i} \in E^{n}\left(x_{i}(A)\right)\right| \exists c^{\prime}: c, e_{1} \hat{\rho}^{n-v}(F) e_{2}^{-1} \preceq c^{\prime}\right\}
\end{aligned}
$$

Consider $\omega \in U_{L(c)}$ and let $n \geq v$. By Lemma 10 there is a unique $\left(a, f, e_{1}\right)$ such that $\omega \in \mathcal{V}_{v}^{n}\left(a, f, e_{1}, e_{1}\right)$. It follows that $e_{1}^{-1} \vdash c$. Now suppose that $\exists e_{2} \in E^{n}(a) \exists c^{\prime}$ : $e_{1} e_{2}^{-1}, c \preceq c^{\prime}$. Then this $e_{2}$ is uniquely determined and $[\omega, c] \in \mathcal{V}_{v}^{n}\left(a, f, e_{1}, e_{2}\right)$. In particular $\mathcal{V}_{v}^{n}\left(a, f, e_{1}, e_{2}\right)$ are for $\left(a, f, e_{1}, e_{2}\right) \in I_{1}^{n}(c)$ pairwise disjoint. If the above assumption is not satisfied then, because of the form $c=u A$, there must be a $(A, F)$ with $a=L(A)$ and $f \preceq L(F)$ such that $\exists e_{2} \in E^{n}\left(x_{2}(A)\right) \exists c^{\prime}: e_{1} \hat{\rho}^{n-v}(F) e_{2}^{-1}, c \preceq$ $c^{\prime}$. Again $e_{2}$ is uniquely determined and $[\omega, c] \in \mathcal{V}_{v}^{n}\left(A, F, e_{1}, e_{2}\right)$. This shows that $\mathcal{V}_{v}^{n}\left(A, F, e_{1}, e_{2}\right)$ are for $\left(A, F, e_{1}, e_{2}\right) \in I_{2 \neq}^{n}(c)$ pairwise disjoint and hence

$$
\mathcal{U}_{c} \subset \bigcup_{I_{1}^{n}(c)}^{\cdot} \mathcal{V}_{v}^{n}\left(a, f, e_{1}, e_{2}\right) \dot{\cup} \bigcup_{I_{2 \neq}^{n}(c)}^{\dot{V}} \mathcal{V}_{v}^{n}\left(A, F, e_{1}, e_{2}\right)
$$

Since for all $(x, y) \in \mathcal{B}_{1}^{v} \cup \mathcal{B}_{2 \neq}^{v}$ and $e_{i} \in E^{n}\left(x_{i}(x)\right), \operatorname{rad}\left(L\left(e_{1} \hat{\rho}^{n-v}(y)\right)\right)$ diverges there is an $n$ such that for all $y$ and all $e_{i}$ either $c \preceq e_{1} \hat{\rho}^{n-v}(y) e_{2}^{-1}$ or $\nexists c^{\prime}: c, e_{1} \hat{\rho}^{n-v}(y) e_{2}^{-1} \preceq c^{\prime}$. In other words, either $\mathcal{U}_{c} \cap \mathcal{V}_{v}^{n}\left(x, y, e_{1}, e_{2}\right)=\mathcal{V}_{v}^{n}\left(x, y, e_{1}, e_{2}\right)$ or that intersection is empty. Thus the inclusion in (83) is an equality for large enough $n$.

By (79), $\mathcal{V}_{v}^{n}\left(a, f, e_{1}, e_{2}\right) \in Q_{v}^{-1}\left(\mathcal{A S G}\left(\mathcal{R}_{\Lambda_{v}}\right)\right)$ so that $\left[\eta\left(\mathcal{V}_{v}^{n}\left(a, f, e_{1}, e_{2}\right)\right)\right]_{\Lambda_{v}}=0$. Thus the first part of the union (33) will not contribute. As for the second, observe that

$$
L\left(\mathcal{V}_{v}^{n}\left(A, F, e_{1}, e_{2}\right)\right)=\left\{\omega \cdot e_{1}^{-1} \mid \omega \in \rho^{n}\left(U_{L(A)}\right) \cap \rho^{n-v}\left(U_{L(F)}\right)\right\}
$$

\footnotetext{
${ }^{2}$ In principle the $v$ below could be chosen different from the one above, but we shall not make use of this generality.
} 
and $\mathcal{V}_{v}^{n}(a, f, e, e)=\left\{\omega \cdot e^{-1} \mid \omega \in \rho^{n}\left(U_{a}\right) \cap \rho^{n-v}\left(U_{f}\right)\right\}$. As above, if $l$ is big enough one gets the alternative

$$
L\left(\mathcal{V}_{v}^{n}\left(A, F, e_{1}, e_{2}\right)\right) \cap \mathcal{V}_{1}^{n+l}(a, f, e, e)=\left\{\begin{array}{l}
L\left(\mathcal{V}_{v}^{n}\left(A, F, e_{1}, e_{2}\right)\right) \\
\emptyset
\end{array}\right.
$$

It follows that (84) (which does not depend on $e_{2}$ ) can be written as a disjoint union

$$
L\left(\mathcal{V}_{v}^{n}\left(A, F, e_{1}, e_{2}\right)\right)=\bigcup_{(a, f, e) \in J^{l}\left(A, F, e_{1}\right)} \mathcal{V}_{1}^{n+l}(a, f, e, e)
$$

for an appropriate $J^{l}\left(A, F, e_{1}\right)$, and moreover, $J^{l}\left(A, F, c e_{1}\right)$ with $R(c)=L\left(e_{1}\right)$ equals to $\left\{(a, f, c e) \mid(a, f, e) \in J^{l}\left(A, F, e_{1}\right)\right\}$. Thus $\left[L\left(\mathcal{V}_{v}^{n}\left(A, F, e_{1}, e_{2}\right)\right)\right]_{\Lambda_{v}}=\left[L\left(\mathcal{V}_{v}^{n}(A, F)\right)\right]_{\Lambda_{v}}$ and we end up with

$$
\left[\eta\left(\mathcal{U}_{c}\right)\right]_{\Lambda_{v}}=\sum_{\left(A, F, e_{1}, e_{2}\right) \in I_{2 \neq}^{n}(c)}\left[\eta\left(V_{v}^{n}(A, F)\right)\right]_{\Lambda_{v}} .
$$

Since $\eta\left(V_{v}^{n}(A, F)\right) \in E_{\mathcal{R}}$ the lemma is proven.

Lemma 13 Let $(a, f) \in \mathcal{B}_{1}^{v},(A, F) \in \mathcal{B}_{2 \neq}^{v}, e \in E^{l}(a), n \geq v$, and $l \geq v$ large enough for the alternative (85) to hold. Then $L\left(\mathcal{V}_{v}^{n}(A, F)\right) \cap \mathcal{V}_{v}^{n+l}\left(a, f, \hat{\rho}^{n}(e), \hat{\rho}^{n}(e)\right) \neq \emptyset$ whenever $L\left(\hat{\rho}^{v}\left(e^{-1}\right) F\right) \preceq \hat{\rho}^{l}(f)$ and $L\left(e^{-1} A\right) \preceq \hat{\rho}^{l-v}(f)$.

Proof:

$$
L\left(\mathcal{V}_{v}^{n}(A, F)\right) \cap \mathcal{V}_{v}^{n+l}\left(a, f, \hat{\rho}^{n}(e), \hat{\rho}^{n}(e)\right) \neq \emptyset
$$

is for $n \geq v$ equivalent to

$$
\left\{\omega \cdot \hat{\rho}^{v}(e) \mid \omega \in \rho^{v}\left(U_{L(A)}\right) \cap U_{L(F)}\right\} \cap \rho^{l+v}\left(U_{a}\right) \cap \rho^{l}\left(U_{f}\right) \neq \emptyset .
$$

That the latter implies $L\left(\hat{\rho}^{v}\left(e^{-1}\right) F\right) \preceq \hat{\rho}^{l}(f)$ and $L\left(e^{-1} A\right) \preceq \hat{\rho}^{l-v}(f)$ is clear provided $l$ is large enough. Now let $(a, f) \in \mathcal{B}_{1}^{v},(A, F) \in \mathcal{B}_{2 \neq}^{v}$, and $\omega \in \rho^{l+v}\left(U_{a}\right) \cap \rho^{l}\left(U_{f}\right)$. Then $L\left(\hat{\rho}^{v}\left(e^{-1}\right) F\right) \preceq \hat{\rho}^{l}(f)$ implies $L(F) \preceq \omega \cdot \hat{\rho}^{v}\left(e^{-1}\right) \in \operatorname{im} \rho^{v}$, and $L\left(e^{-1} A\right) \preceq \hat{\rho}^{l-v}(f)$ implies $L(A) \preceq \rho^{-v}\left(\omega \cdot \hat{\rho}^{v}\left(e^{-1}\right)\right)$.

Define the $\left|\mathcal{B}_{1}^{v}\right| \times\left|\mathcal{B}_{2 \neq}^{v}\right|$ matrices with coefficients $K_{(a, f)(A, F)}^{(l, v)}$ resp. $\mathcal{L}_{(a, f)(A, F)}^{(l, v)}$ through

$$
K_{(a, f)(A, F)}^{(l, v)}:=\left|\left\{e \in E^{l}(a) \mid L\left(\hat{\rho}^{v}\left(e^{-1}\right) F\right) \preceq \hat{\rho}^{l}(f), L\left(e^{-1} A\right) \preceq \hat{\rho}^{l-v}(f)\right\}\right| .
$$

with $l \geq v$ and

$$
\mathcal{L}_{(a, f)(A, F)}^{(l, v)}:=K_{(a, f)(A, F)}^{(l, v)}-K_{(a, f)\left(A^{-1}, F^{-1}\right)}^{(l, v)}
$$

Theorem 4 Let $T$ be a substitution tiling with locally invertible substitution and $\lambda_{v}$ and $\mathcal{L}^{(l, v)}$ as above. If $l$ is large enough so that the alternative (85) holds then, with $k=\left|\mathcal{B}_{1}^{v}\right|$

$$
H(\mathcal{R}) \cong\left\{x \in \mathbb{R}^{k} \mid \exists n \geq 0: \lambda_{v}^{n}(x) \in \mathbb{Z}^{k}\right\} /\left\{x \in \mathbb{R}^{k} \mid \exists n \geq 0: \lambda_{v}^{n}(x) \in \operatorname{im} \mathcal{L}^{(l, v)}\right\} .
$$


Proof: By the last lemma

$$
\left[\chi_{L\left(\mathcal{V}_{v}^{n}(A, F)\right)}\right]_{\Lambda_{v}}=\sum_{(a, f) \in \mathcal{B}_{1}^{v}}\left[\chi_{\mathcal{V}_{v}^{n+l}(a, f)}\right]_{\Lambda_{v}} K_{(a, f)(A, F)}^{(l, v)}
$$

provided alternative (85) holds, and hence

$$
\left[\eta\left(\mathcal{V}_{v}^{n}(A, F)\right)\right]_{\Lambda_{v}}=\sum_{(a, f) \in \mathcal{B}_{1}^{v}}\left[\chi_{\mathcal{V}_{v}^{n+l}(a, f)}\right]_{\Lambda_{v}} \mathcal{L}_{(a, f)(A, F)}^{(l, v)}
$$

As we already saw, $\left[\chi_{\mathcal{V}_{v}^{n}(a, f)}\right]_{\Lambda_{v}}=\left[Q_{v}^{*}\left(\chi_{\xi}\right)\right]_{\Lambda_{v}}$ with $|\xi|=n$ and $r(\xi)=(a, f)$ so that these elements generate $H\left(\mathcal{R}_{\Lambda_{v}}\right)$. Moreover

$$
\left[\chi_{\mathcal{V}_{v}^{n}(a, f)}\right]_{\Lambda_{v}}=\sum_{\left(a^{\prime}, f^{\prime}\right)}\left[\chi_{\mathcal{V}_{v}^{n+1}(a, f)}\right]_{\Lambda_{v}} \lambda_{v}\left(a^{\prime}, f^{\prime}\right)(a, f)
$$

so that

$$
\mathcal{L}^{(l+n, v)}=\lambda_{v}^{n} \mathcal{L}^{(l, v)}
$$

Recall (56) that

$$
H\left(\mathcal{R}_{\Lambda_{v}}\right)=\left\{x \in \mathbb{R}^{k} \mid \exists n \geq 0: \lambda_{v}^{n}(x) \in \mathbb{Z}^{k}\right\} /\left\{x \in \mathbb{R}^{k} \mid \exists n \geq 0: \lambda_{v}^{n}(x)=0\right\} .
$$

The image of $E_{\mathcal{R}} / Q_{v}^{*}\left(E_{\Lambda_{v}}\right)$ in $H\left(\mathcal{R}_{\Lambda_{v}}\right)$ is by Lemma 12 and $(90,92)$ generated by the images of $j_{n} \circ \mathcal{L}^{(l, v)}=\pi \circ \lambda_{v}^{-n} \circ \mathcal{L}^{(l, v)}$. Now $\pi(x) \in \operatorname{im} j_{n} \circ \mathcal{L}^{(l, v)}$ whenever $\lambda_{v}^{n}(x) \in \operatorname{im} \mathcal{L}^{(l, v)}$ for some representative $x$ of $\pi(x)$.

Suppose that $\Omega$ carries an $\mathcal{R}$-invariant (normalized) measure and that the substitution is primitive. Then this measure is unique (and the tiling minimal [5]) and the order structure of $H(\mathcal{R})$ can be expressed as $[x]>0$ for $[x] \in H(\mathcal{R})$ if and only if $\sum_{i} \nu_{i} x_{i}>0$ where $\nu$ is the left-Perron-Frobenius-eigenvector of $\lambda_{v}$. In particular the latter inequality is independent of the chosen representative (the elements of $\operatorname{ker}\left[Q_{v}^{*}\right]$ are infinitesimal).

\subsubsection{Simplifications}

The determination of $\mathcal{L}^{(l, v)}$ can be quite cumbersome. But simplifications occurr under certain circumstances.

1) In case that for all $(a, f) \in \mathcal{B}_{1}^{v}, a$ is uniquely determined by $f$ (we write it as $a(f)$ ) $L\left(\hat{\rho}^{v}\left(e^{-1}\right) F\right) \preceq \hat{\rho}^{l}(f)$ implies $L\left(e^{-1} A(F)\right) \preceq \hat{\rho}^{l-v}(f)$. Thus $K^{(l, v)}$ simplifies to

$$
K_{f F}^{(l, v)}:=K_{(a, f)(A, F)}^{(l, v)}=\left|\left\{e \in E^{l}(a(f)) \mid L\left(\hat{\rho}^{v}\left(e^{-1}\right) F\right) \preceq \hat{\rho}^{l}(f)\right\}\right| .
$$

This is for instance the case if $v=0$.

2) In some sense the other extreme is that the $f$ is determined by $a$. This is case if the substitution forces its border. It has been partly analyzed in [5]. In the present notation it is expressed as follows:

Definition 14 A locally invertible substitution forces its border if there is a $v$ such that $\beta_{v}: \mathcal{B}_{1}^{v} \rightarrow \mathcal{M}_{\mathbb{I} 1}$ is a bijection. 
Not only the graph simplifies enormously, in that it coincides with the improper graph, but also we may take $l=v$. Moreover $L\left(e^{-1} A\right) \preceq \hat{\rho}^{l-v}(f(a))$ implies $L\left(\hat{\rho}^{v}\left(e^{-1}\right) F(A)\right) \preceq$ $\hat{\rho}^{l}(f(a))$ where we wrote $f(a)$ for the $f$ determined by $a$. Hence for substitutions which force their border

$$
K_{a A}^{(v, v)}:=K_{(a, f)(A, F)}^{(v, v)}=\left|\left\{e \in E^{v}(a) \mid L\left(e^{-1} A\right) \preceq f(a)\right\}\right| .
$$

Note that a substitution can force its border only for $v \geq 1$, because otherwise any pointed tiling would be determined by its pointed tile and hence periodic which contradicts local invertibility.

As for the determination of $H(\mathcal{R})$ we have:

3) If $\lambda_{v}$ is invertible over $\mathbb{Z}$ then $H(\mathcal{R})=\mathbb{Z}^{k} /\left\langle\bigcup_{n} \lambda_{v}^{-n} \operatorname{im} \mathcal{L}^{(l, v)}\right\rangle$.

\subsection{Examples}

The above machinerie has been designed to tackle higher dimensional tilings. We therefore will present the computation of the coinvariants associated to the Penrose tilings. But it is also applicable to one dimensional substitutions in the sense of [6]. To allow the reader a comparison with technics used elsewhere to obtain the integer group of coinvariants, c.f. 8, 9, we present the Thue Morse substitution as an example.

\subsubsection{The Thue Morse substitution}

The Thue Morse substitution $\varrho$ is defined on the two letter alphabet $\{a, b\}$ by

$$
\begin{aligned}
a & \mapsto a b \\
b & \mapsto b a
\end{aligned}
$$

and extended to words as $\varrho\left(a_{1} \ldots a_{k}\right)=\varrho\left(a_{1}\right) \ldots \varrho\left(a_{k}\right)$. It may be viewed (like any other one dimensional substitution of the kind in [6]) as a substitution in the algebraic sense of the tiling which is a fixed point under $\varrho$ : Consider the sequence over $\mathbb{Z}^{\geq 0}$ with values in $\{a, b\}$ given by $\varrho^{\infty}(a)$ and complete it to a sequence over $\mathbb{Z}$ by reflection (i.e. by $\varrho^{2 \infty}(a)$ to the left). This represents a one dimensional pointed tiling the pointed tile (letter) being the one on 0 , i.e. the first one of (the right) $\varrho^{\infty}(a)$. The (finite) words appearing in $\varrho^{\infty}(a)$ with two letters chosen are doubly pointed pattern classes. . $^{\beta}$ We indicate the first by a grave and the second by an acute, and if both coincide

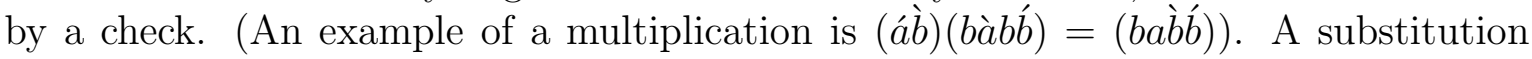
as a homomorphism of the almost-groupoid of doubly pointed pattern classes is then given by $\hat{\rho}\left(w_{1} \grave{a}_{i} w_{2} \hat{a}_{j} w_{3}\right)=\varrho\left(w_{1}\right) \grave{a}_{i_{1}} a_{i_{2}} \varrho\left(w_{2}\right) \hat{a}_{j_{1}} a_{j_{2}} \varrho\left(w_{3}\right)$ where we have used the writing $\varrho\left(a_{i}\right)=a_{i_{1}} a_{i_{2}}$.

The substitution is locally invertible but does not force its border and there is no advantage in using large $v$. We therefore take $v=0$. The connectivity matrix of $\Sigma_{0}$ is

$$
\lambda_{0 f_{1} f_{2}}=\text { number of } e \in E^{1}\left(a\left(f_{1}\right)\right) \text { with } f_{2} \preceq L\left(e \check{\rho}\left(f_{1}\right)\right) \text {. }
$$

\footnotetext{
${ }^{3}$ If we do not have a geometric interpretation of the tilings as sequences of decorated intervals we have to restrict to pattern classes which are connected.
} 
We have

$$
\begin{gathered}
\mathcal{B}_{1}^{0}=\{a \check{a} b, b \check{a} a, b a \check{b} b, b \check{b} a, a \check{b} b, a \check{b} a\} \\
\hat{\rho}\left(\mathcal{B}_{1}^{0}\right)=\{a b \check{a} b b a, b a \check{a} b a b, b a \check{a} b b a, b a \check{b} a a b, a b \check{b} a b a, a b \check{b} a a b\} \\
E^{1}(a)=\{\check{a} b, \dot{a} \grave{b}\} \quad E^{1}(b)=\{\check{b} a, b \grave{b}\}
\end{gathered}
$$

Taking the elements of $\mathcal{B}_{1}^{0}$ and $\hat{\rho}\left(\mathcal{B}_{1}^{0}\right)$ in the above order one obtains

$$
\lambda_{0}=\left(\begin{array}{llllll}
0 & 0 & 1 & 0 & 1 & 0 \\
1 & 0 & 0 & 0 & 0 & 1 \\
1 & 0 & 0 & 0 & 1 & 0 \\
0 & 1 & 0 & 0 & 0 & 1 \\
0 & 0 & 1 & 1 & 0 & 0 \\
0 & 1 & 0 & 1 & 0 & 0
\end{array}\right)
$$

The Perron-Frobenius-eigenvalue is 2 and the normalized left-Perron-Frobenius-eigenvector is $\nu=\frac{1}{6}(1,1,1,1,1,1)$. Hence

$$
\mu(H(\mathcal{R}))=\frac{1}{3} \mathbb{Z}\left[\frac{1}{2}\right]
$$

To obtain the full group we may split up $\mathcal{B}_{2 \neq}^{0}=\mathcal{B}_{2<}^{0} \cup\left(\mathcal{B}_{2<}^{0}\right)^{-1}$ with

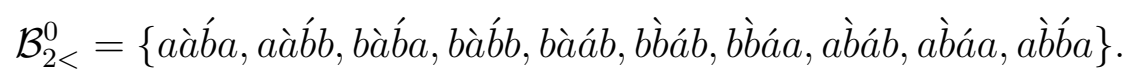

Thus (85) holds for $l=1$, i.e. we have to determine

$$
K_{f F}^{(1,0)}=\text { number of } e \in E^{1}(a(f)) \text { with } L(F) \preceq L(e \hat{\rho}(f)) .
$$

Let $\mathcal{L}_{<}^{(1,0)}$ be the restriction of $\mathcal{L}^{(1,0)}$ to indices $f F$ with $F \in \mathcal{B}_{2<}^{0}$. Then, again with respect to the above order,

$$
\mathcal{L}_{<}^{(1,0)}=\left(\begin{array}{cccccccccc}
0 & 0 & 0 & 0 & 0 & 0 & 0 & -1 & 0 & 1 \\
0 & 0 & 0 & 0 & -1 & 0 & 0 & 1 & 0 & 0 \\
0 & 0 & 0 & 0 & -1 & 0 & 0 & 0 & 0 & 1 \\
0 & 0 & -1 & 0 & 1 & 0 & 0 & 0 & 0 & 0 \\
0 & 0 & 1 & 0 & 0 & 0 & 0 & 0 & 0 & -1 \\
0 & 0 & 0 & 0 & 1 & 0 & 0 & 0 & 0 & -1
\end{array}\right)
$$

And, since $\mathcal{L}_{f F^{-1}}^{(1,0)}=-\mathcal{L}_{f F}^{(1,0)}, \operatorname{im} \mathcal{L}^{(1,0)}=\operatorname{im} \mathcal{L}_{<}^{(1,0)} \cong \mathbb{Z}^{3} . \lambda_{0} \mathcal{L}_{<}^{(1,0)}$ is up to a permutation of the columns $\mathcal{L}_{<}^{(1,0)}$, i.e. $\lambda_{0}$ preserves $\operatorname{im} \mathcal{L}^{(1,0)}$. In fact, $\lambda_{0}$ is diagonalizable, it has eigenvalues $2,1,0,-1,-1$ occurring with multiplicity 3 , and $\operatorname{im} \mathcal{L}^{(1,0)}$ is spanned by the (right) eigenvector to eigenvalue 1 together with a two dimensional subspace of the eigenspace to eigenvalue -1 . A system of (right) eigenvectors is given by

$$
\left(\begin{array}{l}
1 \\
1 \\
1 \\
1 \\
1 \\
1
\end{array}\right) \quad\left(\begin{array}{c}
-1 \\
0 \\
-1 \\
1 \\
0 \\
1
\end{array}\right) \quad\left(\begin{array}{c}
-1 \\
-1 \\
-1 \\
1 \\
1 \\
1
\end{array}\right) \quad\left(\begin{array}{c}
0 \\
-1 \\
-1 \\
0 \\
1 \\
1
\end{array}\right) \quad\left(\begin{array}{c}
-1 \\
1 \\
0 \\
-1 \\
1 \\
0
\end{array}\right) \quad\left(\begin{array}{c}
1 \\
-1 \\
-1 \\
1 \\
0 \\
0
\end{array}\right)
$$


It follows that

$$
H(\mathcal{R}) \cong \mathbb{Z}\left[\frac{1}{2}\right] \oplus \mathbb{Z}
$$

The elements of the second summand are infinitesimal since the pairing between the left Perron-Frobenius-eigenvector $\nu$ and all vectors from the (right) eigenspace to eigenvalue -1 is zero. Thus the positive cone is $\mathbb{Z}\left[\frac{1}{2}\right]^{+}$.

\subsubsection{Penrose tilings}

There are several well known variants of tilings which are called Penrose tilings [27, 11] and which are a priori to be distinguished as they lead to non-isomorphic groupoids. But they may be transformed into each other by purely local manipulations which implies that one can find maps satisfying the conditions of Lemma 6 and leading to isomorphisms between reductions of the corresponding groupoids. Since any such tiling is minimal all reductions lead to the same ordered integer group of coinvariants differing possibly in the order unit. The version which is most suitable for our purposes is the one which has triangles as tiles, cf. Figure 7. The triangles are decorated (with a little circle) to break the mirror symmetry. There are 40 pattern classes of them. The orientational symmetry of a tiling (or its class) is the largest subgroup of $O(d)$, acting on a pointed tiling being identified with a representative in $\mathbb{R}^{d}$ in the obvious way, which leaves the hull invariant. The orientational symmetry of a Penrose tiling by triangles possesses 20 elements. It is generated by a rotation around $\frac{\pi}{5}$ together with a mirror reflection at a boundary line of a triangle [5]. We denote by $\mathcal{M}_{\mathbb{I}}$ resp. $\mathcal{R}$ the almost-groupoid resp. groupoid associated to these tilings. The well known deflation of these tilings $\rho_{t}$ with $t=\frac{1+\sqrt{5}}{2}$ is displayed below. Since it is covariant with respect to the orientational symmetry it suffices to give it for one orientation only.

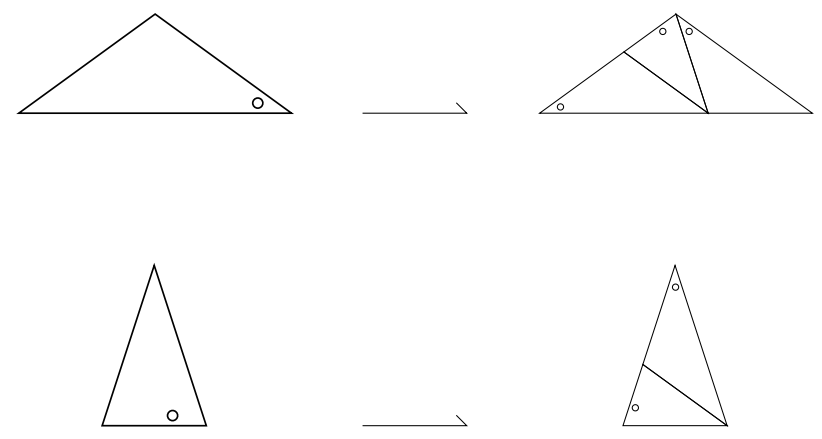

A replacement $\rho_{t}(a)$ taking exactly the same space as the tile $a$ the relative position measured with respect to the centers of gravity is 0 . Choosing pointed tiles for $\rho_{t}(a)$ we obtain a substitution $\hat{\rho}$. Those tilings which have an exact five-fold symmetry are invariant under $\hat{\rho}^{4}$. The substitution is primitive, locally invertible, and forces its border with $v=4$ [5]. Hence $\Lambda_{4}=\Sigma$ and $\lambda_{4}=\sigma$, the substitution matrix of $\hat{\rho}$. Since $\sigma$ is invertible over the integers $H\left(\mathcal{R}_{\Sigma}\right) \cong \mathbb{Z}^{40}$. To simplify the computation of $E_{\mathcal{R}} / Q_{4}^{*}\left(E_{\Sigma}\right)$ we make use of the symmetry properties of the tiling and in particular 
of the fact that the boundaries of substitutes $\hat{\rho}^{4}(a)$ are local mirror axes so that the pattern classes of those $A \in \mathcal{M}_{\mathbb{I} 2, \neq}$ which cross the boundaries of 4 -fold substitutes are always mirror symmetric [5], c.f. below where the boundaries are indicated through fatter lines.

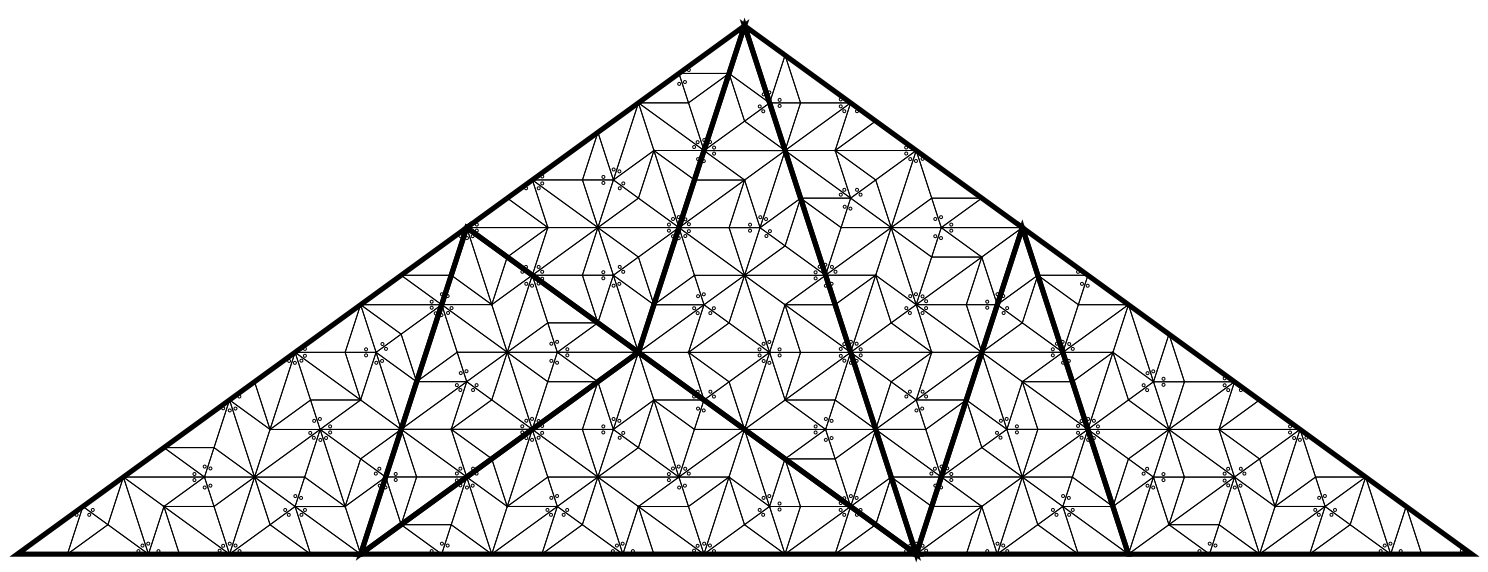

Let $\alpha$ denote the direction of a boundary line of a tile resp. a substitute. There are 10 different ones and we order them anti-clockwise identifying them with $\{0, \cdots, 9\}$. Saying that a substitute has boundary $\alpha$ if it has a boundary with that direction we define the $40 \times 40$ matrices with entries

$$
N_{a b}^{\alpha}:=\text { number of } a \text { 's at boundary } \alpha \text { of } \hat{\rho}^{4}(b) .
$$

In particular $N_{a b}^{\alpha}=0$ in case $a$ or $\rho(b)$ do not have boundary $\alpha$. Let $\alpha(a)$ be the mirror image of $a$ with respect to the mirror axis $\alpha$ and define

$$
\mathcal{D}_{a b}^{\alpha}=N_{a b}^{\alpha}-N_{a \alpha(b)}^{\alpha} .
$$

Then

$$
\mathcal{L}_{a A}^{(4,4)}=\left\{\begin{array}{cl}
\mathcal{D}_{a b}^{\alpha} & \text { if }|A|=b \alpha(b) \text { and } x(A)=b \\
-\mathcal{D}_{a b}^{\alpha} & \text { if }|A|=b \alpha(b) \text { and } x(A)=\alpha(b) \\
0 & \text { else }
\end{array}\right.
$$

$|A|=b \alpha(b)$ indicating that the pattern class of $A$ is composed of $b$ and $\alpha(b)$ in such a way that the common boundary is the symmetry axis. Hence $\operatorname{im} \mathcal{L}^{(4,4)}=$ $\left\langle\operatorname{im} \mathcal{D}^{\alpha}, \alpha=0, \ldots, 9\right\rangle$. $\mathcal{D}^{\alpha}$ is related to $\mathcal{D}^{0}$ by symmetry, i.e. $\mathcal{D}^{\alpha}=R^{-\alpha} \mathcal{D}^{0} R^{\alpha}, R$ being the matrix which acts as a rotation around $\frac{\pi}{5}$.

To be very explicit let us use a basis $\left\{e_{10 k+\alpha}\right\}_{0 \leq k \leq 3,0 \leq \alpha \leq 9}$ of $H\left(\mathcal{R}_{\Sigma}\right)$ with $e_{10 k+\alpha}=$ $\left[\chi_{U_{a_{10 k+\alpha}}}\right]_{\Sigma}$ where $a_{10 k+\alpha}$ corresponds to the pattern class of the triangle in Figure $7 . \mathrm{k}$ rotated around an angle of $\frac{\alpha \pi}{5}$.

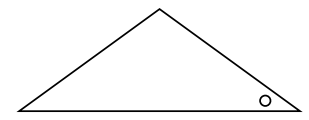

Figure 7.0

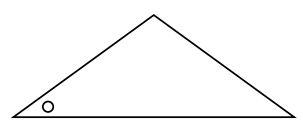

Figure 7.1

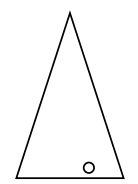

Figure 7.2

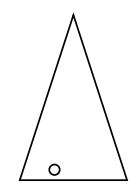

Figure 7.3 
In terms of the rotation matrix $\omega$, which has entries $\omega_{\alpha \beta}=\delta_{\alpha-\beta, 1 \bmod 10}, R$ and the substitution matrix $\sigma$ are given by

$$
R=\left(\begin{array}{cccc}
\omega & 0 & 0 & 0 \\
0 & \omega & 0 & 0 \\
0 & 0 & \omega & 0 \\
0 & 0 & 0 & \omega
\end{array}\right), \quad \sigma=\left(\begin{array}{cccc}
\omega^{4} & \omega^{0} & 0 & \omega^{6} \\
\omega^{0} & \omega^{6} & \omega^{4} & 0 \\
\omega^{3} & 0 & \omega^{7} & 0 \\
0 & \omega^{7} & 0 & \omega^{3}
\end{array}\right)
$$

The matrix $N^{0}$ may be read of from Figure 8 (and completed by symmetry); it is given below (108). Moreover $\mathcal{D}^{0}=N^{0}-N^{0} S$ where $S$ implements the reflection at $\alpha=0$, explicitly, with $s_{\alpha \beta}=\delta_{\alpha+\beta, 5 \bmod 10}$ (counting rows and columns form 0 to 9 )

$$
S=\left(\begin{array}{cccc}
0 & s & 0 & 0 \\
s & 0 & 0 & 0 \\
0 & 0 & 0 & s \\
0 & 0 & s & 0
\end{array}\right)
$$

It turns out that $\operatorname{im} \mathcal{L}^{(4,4)}$ is generated by the orbit under $R$ of (the transpose of) the four vectors

$$
\begin{gathered}
v_{1}=(0,0,0,0,0,0,0,0,0,0,0,0,0,0,0,0,0,0,0,0,0,0,0,0,0,0,1,0,0,0,0,-1,0,0,0,0,0,0,0,0) \\
v_{2}=(0,0,0,0,0,0,1,0,0,0,0,-1,0,0,0,0,0,0,0,0,0,0,0,0,0,0,0,0,0,0,0,0,0,0,0,0,0,0,0,0) \\
w_{1}=(0,0,0,0,0,0,0,1,0,0,-1,0,0,0,0,0,0,0,0,0,0,0,0,0,0,0,0,0,1,0,0,0,0,0,0,0,0,0,0,-1) \\
w_{2}=(0,0,0,0,0,1,0,0,0,0,0,0,-1,0,0,0,0,0,0,0,0,0,0,0,0,0,0,0,0,-1,0,0,0,0,0,0,0,0,1,0)
\end{gathered}
$$

but 8 of the 40 vectors thus obtained are linearly dependent of the others. Moreover $\operatorname{im} \mathcal{L}^{(4,4)}$ is invariant under $\sigma$. Dividing it out yields no torsion so that we obtain

$$
H(\mathcal{R}) \cong \mathbb{Z}^{8}
$$

To obtain the order structure we look for the smallest subgroup $I$ of $\mathbb{Z}^{40}$ which is invariant under $\sigma$ and spans a real vector space containing the right Perron-Frobeniuseigenvector of $\sigma$. Then the positive cone of $\mathbb{Z}^{40}$ is given by those $x \in I$ which pair with the left-Perron-Frobenius-eigenvector $\nu$ of $\sigma$ to $\nu x>0$. All other elements must pair with $\nu$ to zero and are thus infinitesimal. Clearly $I$ is spanned by (the transpose of)

$$
x_{1}=(\overbrace{1, \ldots, 1}^{20}, \overbrace{0, \ldots, 0}^{20}), \quad x_{2}=(\overbrace{0, \ldots, 0}^{20}, \overbrace{1, \ldots, 1}^{20})
$$

and $\nu$ is given by $\nu=\frac{2-t}{20}\left(t x_{1}+x_{2}\right)^{T}$ (in its normalized form). Thus $I \cap \operatorname{im} \mathcal{L}^{(4,4)}=\emptyset$ and

$$
H(\mathcal{R}) \cong I \oplus \mathbb{Z}^{6}
$$

the elements of the second summand being infinitesimal. The range of the unique state on $H(\mathcal{R})$, which of course coincides with that on $H\left(\mathcal{R}_{\Sigma}\right)$, is

$$
\mu(H(\mathcal{R}))=\frac{1}{20}(\mathbb{Z}+t \mathbb{Z}) .
$$


This concludes the computation of the integer group of coinvariants and the range of its state for the Penrose tilings. But it may be instructive to look at result (102) from a different point of view. Let $H_{1}$ be the sublattice of $\mathbb{Z}^{40}$ which is generated by $\left\{R^{\alpha} v_{i}\right\}_{i=1,2 ; \alpha=0,1 \cdots, 9} \cdot H\left(\mathcal{R}_{\Sigma}\right) / H_{1}=\mathbb{Z}^{20}$ has a basis with natural geometrical interpretation. It is formed by the classes of characteristic functions on the pattern classes of rhombi which are always formed by either two smaller triangles or two larger triangles. This indicates that one should look at the Penrose tilings by rhombi. Such a tiling, which we denote by $T_{R h}$, has only 20 pattern classes of tiles. That the groupoid associated to the Penrose tilings by rhombi is isomorphic to a reduction of $\mathcal{R}$ may be seen as follows: Let $\mathcal{C} \subset \mathcal{M}_{\mathbb{I} 1}$ consist of the classes of triangles that are obtained from those of Figure 7.1 and 7.3 by a rotation. Let $R h \subset \mathcal{M}_{\mathbb{I I}}$ be the almost-groupoid given by elements of the form $a c a^{\prime}$ where $a, a^{\prime} \in \mathcal{C}$ and $c \in \mathcal{M}_{\mathbb{I}}$ is such that all its triangles pair to rhombi. Clearly $R h$ is approximating generating and regular. Now deleting the diagonal which coincides with the base of the two touching triangles yields an isomorphism of almost-groupoids $R h \rightarrow \mathcal{M}_{\mathbb{I}}\left(T_{R h}\right)$ which satifies the growth condition with $t=1$. Hence the groupoid associated to the rhombus version is isomorphic to $\mathcal{R}_{R h}$.

The largest subgroup of $O(2)$ leaving $\Omega_{R h}$ invariant consists only of the rotations which are multiples of $\frac{\pi}{5}$ since the mirror image of a $\omega \in \Omega_{R h}$ lies in $\Omega \backslash \Omega_{R h}$. However, to our knowledge there is no substitution for Penrose tilings by rhombi which is covariant even under this reduced orientational symmetry. But there are non covariant ones, namely 10 of them, the deflation corresponding to $\hat{\rho}_{0}$ being given in Figure 9 and $\hat{\rho}_{\alpha}$ being obtained from $\hat{\rho}_{0}$ just by rotation of the whole figure around $\frac{\alpha \pi}{5}, \alpha=0, \cdots, 9$. (The relative positions are indicated by a cross.) That all these substitutions are primitive locally invertible and force their border carries over from $\hat{\rho}$. It turns out that

$$
\operatorname{ker} \sigma_{\alpha}=\left\langle\left\{\left[R^{\alpha} w_{i}\right]_{H_{1}}\right\}_{i=1,2}\right\rangle
$$

where $\sigma_{\alpha}$ is the substitution matrix of $\hat{\rho}_{\alpha}$ and $[\cdot]_{H_{1}}$ denotes the classes in $H\left(\mathcal{R}_{\Sigma}\right) / H_{1}$. Since the restriction of $\sigma_{\alpha}$ to its image is an automorphism and $\operatorname{ker} \sigma_{0}=\operatorname{ker} \sigma_{0}^{2}$ we have

$$
H\left(\mathcal{R}_{\Sigma_{\alpha}}\right) \cong H\left(\mathcal{R}_{\Sigma}\right) /\left\langle\left\{H_{1}, R^{\alpha} w_{i}, i=1,2\right\}\right\rangle
$$

$\Sigma_{\alpha}$ denoting the graph having $\sigma_{\alpha}$ as connectivity matrix. Any of the substitutions $\hat{\rho}_{\alpha}$ leads to a homeomorphism $Q_{\alpha}$ between $\Omega_{R h}$ and the path space $\mathcal{P}_{\Sigma_{\alpha}}$ and to a surjective homomorphism $\left[Q_{\alpha}^{*}\right]: H\left(\mathcal{R}_{\Sigma_{\alpha}}\right) \rightarrow H(\mathcal{R})$. Let $\pi_{\alpha}: H\left(\mathcal{R}_{\Sigma}\right) \rightarrow H\left(\mathcal{R}_{\Sigma_{\alpha}}\right)$ be the natural projection. Then $\left[Q_{4}^{*}\right]=\left[Q_{\alpha}^{*}\right] \circ \pi_{\alpha}$ for all $\alpha$ and therefore

$$
\operatorname{ker}\left[Q_{4}^{*}\right] \supset\left\langle\left\{H_{1}, R^{\alpha} w_{i}, i=1,2 ; \alpha=1, \cdots, 9\right\}\right\rangle \cong \mathbb{Z}^{32} .
$$

This shows independently that $H(\mathcal{R}) \subset \mathbb{Z}^{8}$ but not the opposite inclusion. A computation of $\operatorname{im} \mathcal{L}^{(4,4)}$ for e.g. $\rho_{0}$ would have been more complicated due to the lack of symmetry.

Connes associates to the Penrose tilings yet another graph, the folded $A_{4}$ Coxeter graph, making use of a coding of a tiling by 0,1 sequences obeying the condition that no consecutive 1's can appear [12]. But this coding, which was found by Robinson [11, does not distinguish between a tiling and its image under an element of the orientational symmetry. In fact, the coding yields a homeomorphism between the hull 
modulo the orientational symmetry and the path space of the folded $A_{4}$ graph, with the effect that the groupoid arising is that given by cofinality. In other words, the tilings are considered as equivalence classes under translations and rotations and reflections. One then obtains an $A F$-algebra as groupoid- $C^{*}$-algebra whose $K_{1}$-group is trivial and whose $K_{0}$-group coincides with the integer group of coinvariants of the groupoid and may be identified with the group $I$ in (103) as ordered group. Although the range of the tracial state on it coincides with (104) up to the order unit (the factor $\frac{1}{20}$ does not appear) it is a priori not clear that it predicts the right gap labels since the $A F$-algebra does not contain the discrete Laplacian.

\section{Concluding Questions}

We have computed the integer group of coinvariants and its order but even in two dimensions and under the assumption that $K_{0}\left(\mathcal{A}_{T}\right)=H(\mathcal{R}) \oplus \mathbb{Z}$ it is not clear what the order structure on the $K_{0}$-group is.

We did not mention groupoid cohomology but at least for tilings which reduce to decorations the integer group of coinvariants is a cohomology group of the groupoid $\mathcal{R}$ with coefficients in $\mathbb{Z}$. The result of $\llbracket$ on the connection between $K$-theory and group cohomology is easily seen to generalize to this situation since the stability of $K$-theory under taking stably isomorphic algebras is mirrored by the stability of (continuous) groupoid cohomology under taking (continuously) similar groupoids. It is therefore

tempting to believe that $K$-theory of algebras associated to tilings is always related to the groupoid cohomology with coefficients in $\mathbb{Z}$ of the associated groupoid in a way

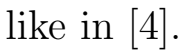




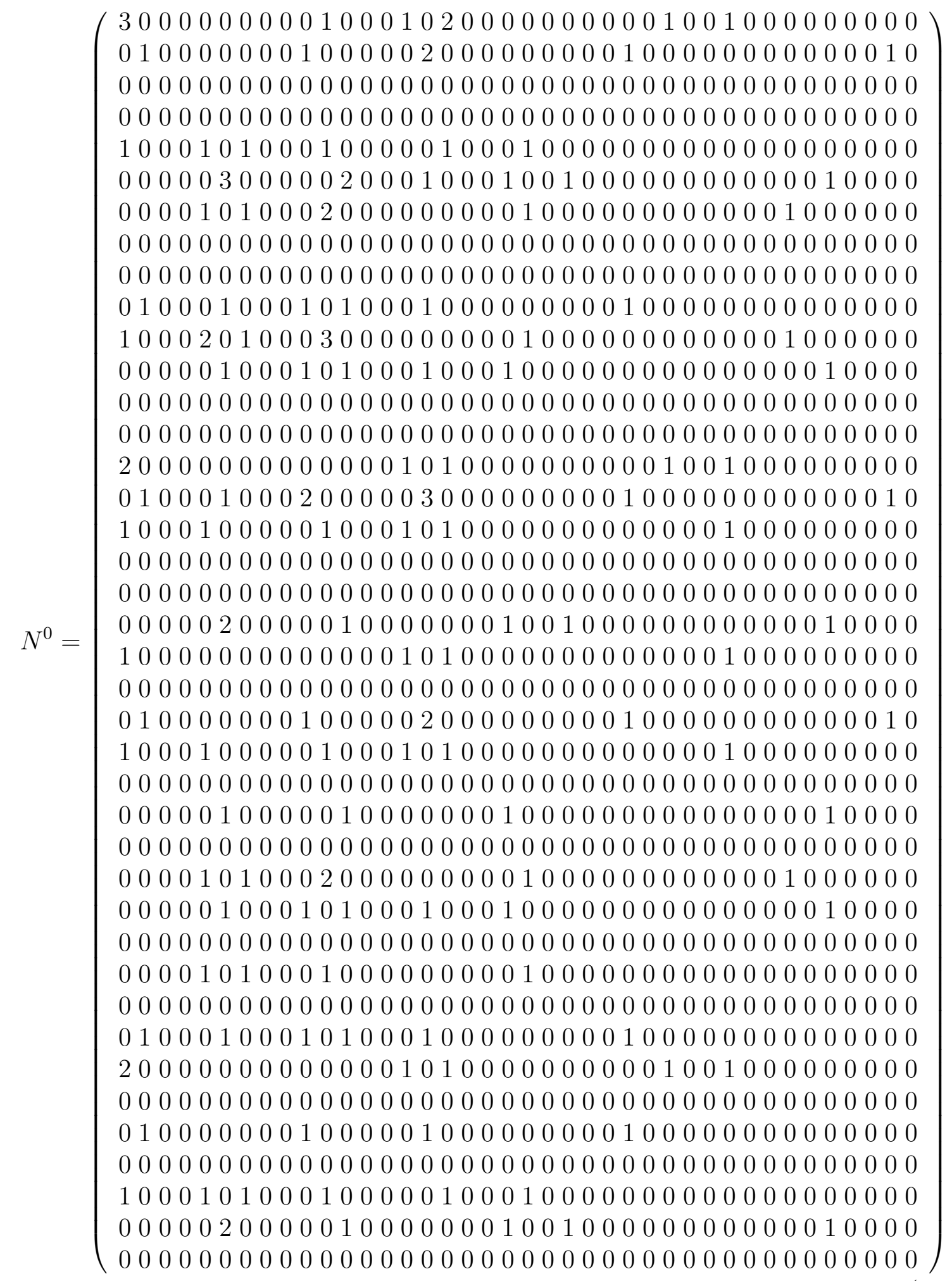

(108) 

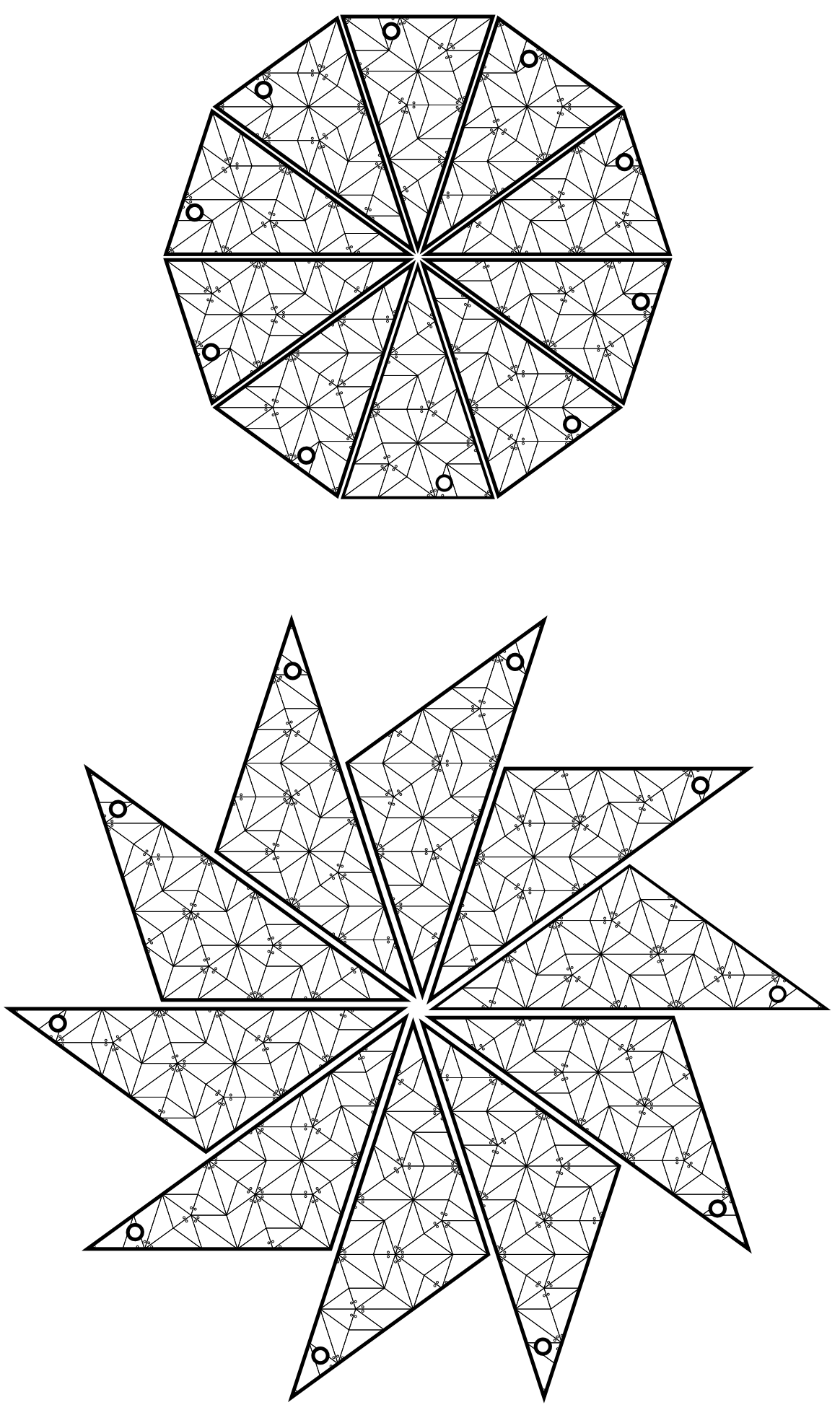

Figure 8: 4-fold substitutes of the triangles of Figure 7.0 and 7.1 in all directions. 

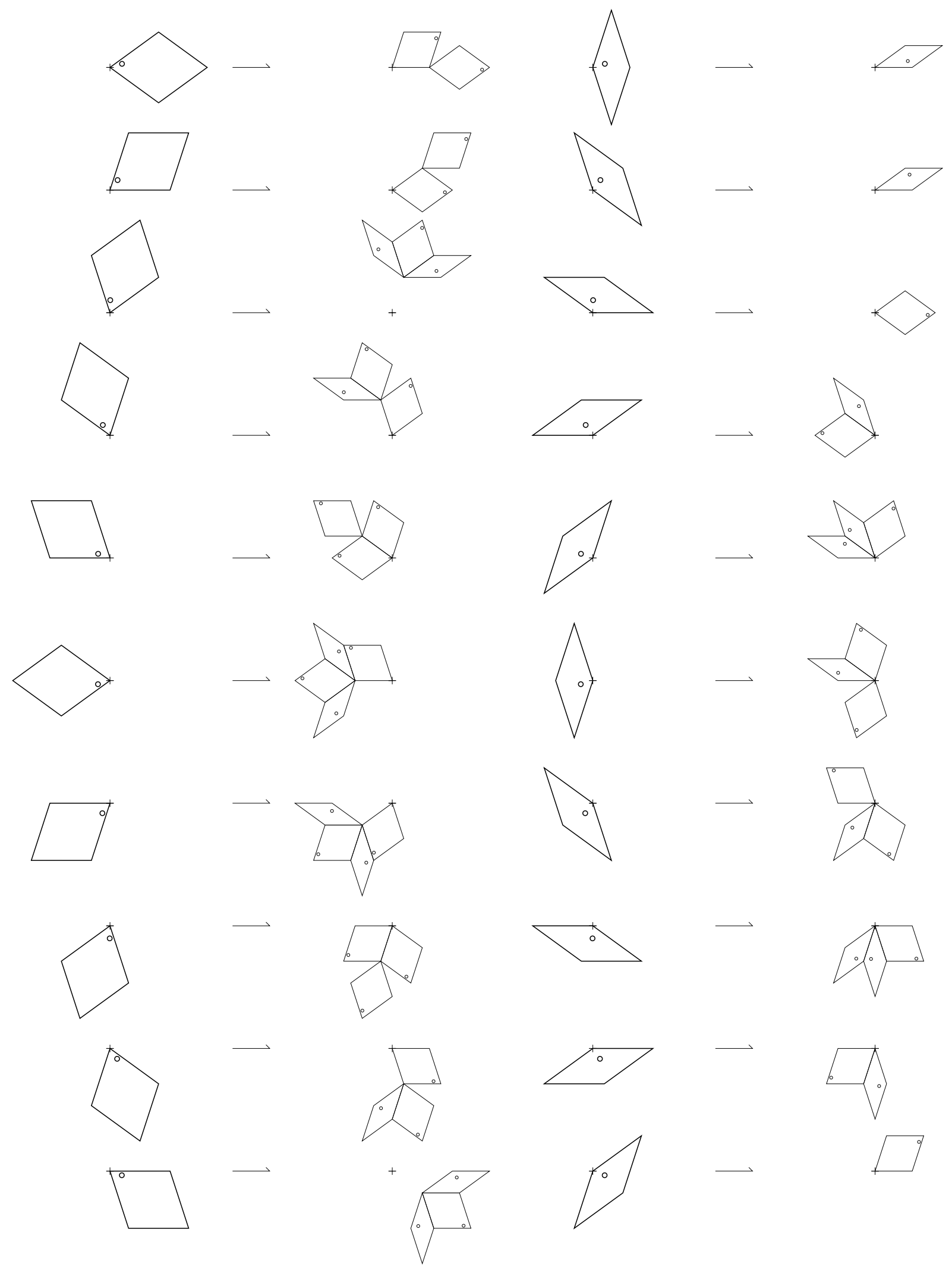

Figure 9 


\section{References}

[1] J. Bellissard. Schrödinger's operators with an almost periodic potential. In R. Schrader and R. Seiler, editors, Mathematical Problems in Theoretical Physics. Lecture Notes in Physics 153:356-359, Springer Verlag, 1982.

[2] R.H. Herman, I.F. Putnam, and C.F. Skau. Ordered Bratteli diagrams, dimension groups and topological dynamics. Int. J. Math., 3:827-864, 1992.

[3] T. Giordano, I.F. Putnam, and C.F. Skau. Topological orbit equivalence and $C^{*}$-crossed products. Trondheim preprint Mathematics No. 11/1993, 1993.

[4] A.H. Forrest and J. Hunton. The cohomology and K-theory of commuting homeomorphisms of the cantor set. Edinburgh preprint MS95-024, 1995.

[5] J. Kellendonk. Non commutative geometry of tilings and gap labelling. King's College London preprint KCL-TH-94-1. To appear in Rev. Math. Phys.

[6] M. Queffélec. Substitution Dynamical Systems. Lect. Notes in Math. 1294. Springer-Verlag, 1987.

[7] J. Bellissard, A. Bovier, and J.M. Ghez. Gap labelling theorems for one dimensional discrete Schrödinger operators. Rev. Math. Phys., 4:1-38, 1992.

[8] A.H. Forrest. K-groups associated with substitution minimal systems. To appear in Israel J. Math.

[9] B. Host. Dimension groups of substitution dynamical systems. Marseille, unpublished course notes, 1993.

[10] J. Renault. A Groupoid approach to $C^{*}$-Algebras. Lect. Notes in Math. 793. Springer-Verlag, 1980.

[11] B. Grünbaum and G.C. Shephard. Tilings and Patterns. Freeman and Company (New York), 1987.

[12] A. Connes. Non Commutative Geometry. Academic Press, 1994.

[13] A. Ramsey. Virtual groups and group actions. Advances in Math., 6:253-322, 1971.

[14] M. Baake, M. Schlottmann, and P.D. Jarvis. Quasiperiodic tilings with tenfold symmetry and equivalence with respect to local derivability. J. Phys. A, 24:46374654, 1991.

[15] J.E.S. Socolar, P.J. Steinhardt, and D. Levine. Quasicrystals with arbitrary orientational symmetry. Phys. Rev. B, 32:5547-5550, 1985.

[16] M. Duneau and A. Katz. Quasiperiodic patterns and icosahedral symmetry. J. Physique, 47:181-196, 1986. 
[17] L.G. Brown. Stable isomorphism of hereditary subalgebras of $C^{*}$-algebras. Pacific J. of Math., 71:335-348, 1977.

[18] J. Bellissard. K-Theory of $C^{*}$-Algebras in Solid State Physics. In T.C. Dorlas, N.M. Hugenholtz, and M. Winnik, editors, Statistical Mechanics and Field Theory: Mathematical Aspects. Lect. Notes in Phys. 257:99-156, Springer Verlag, 1986.

[19] B. Blackadar. K-Theory for Operator Algebras. MSRI Publication 5. Springer Verlag, 1986.

[20] G.J. Murphy. $C^{*}$-Algebras And Operator Theory. Academic Press, 1990.

[21] M.A. Shubin. Discrete magnetic Laplacian. Commun. Math. Phys., 164:259-275, 1994.

[22] G. Pedersen. $C^{*}$-Algebras and their Automorphism Groups. Academic Press, London/NewYork/San Francisco, 1979.

[23] A. van Elst. Gap-labelling theorems for Schrödinger operators on the square and cubic lattice. Rev. Math. Phys., 6:319-342, 1994.

[24] G. Skandalis. Kasparov's bivariant $K$-theory and applications. Preprint

[25] E.G. Effros. Dimensions and $C^{*}$-Algebras. Conference Board Math. Sci. 46. Amer. Math. Soc. (Providence R.I.), 1981.

[26] S. Lang. Algebra. Addison-Wesley, 1984.

[27] R. Penrose. The rôle of aesthetics in pure and applied mathematical research. Bull. Inst. Math. Appl., 10(7/8):266-71, 1974. 


\section{Contents}

$\begin{array}{ll}\text { Introduction } & 2\end{array}$

\begin{tabular}{|lr}
\hline Tilings and groupoids & 4
\end{tabular}

1.1 Almost-groupoids . . . . . . . . . . . . . . . . . . . . . . . 4

1.2 Tilings . . . . . . . . . . . . . . . . . . . . 5

1.2 .1 The integer group of coinvariants associated to a tiling . . . . . 10

1.3 Reduction . . . . . . . . . . . . . . . . . . . 10

1.4 Locally defined maps between tilings . . . . . . . . . . . . . . . . . . . 12

1.5 Decorations of $\mathbb{Z}^{d} \ldots \ldots \ldots \ldots \ldots$. . . . . . . . . . . . . . . . . . . 14

\begin{tabular}{|ll|l}
2 & $C^{*}$-algebraic characterization of tilings & 18
\end{tabular}

$2.1 \quad K$-theoretical gap labelling . . . . . . . . . . . . . . . . . . . . . . . . . 19

$2.2 \quad K_{0 \text {-groups for tilings } \ldots \ldots \ldots \ldots} \ldots \ldots \ldots \ldots$

3 Substitution tilings 23

3.1 Deflation . . . . . . . . . . . . . . . . . . . . . 23

3.2 Path spaces over graphs and their dimension groups . . . . . . . . . . . 24

3.3 Path spaces determined by substitutions . . . . . . . . . . . . . . . . . 26

3.4 The integer group of coinvariants for substitution tilings . . . . . . . . 30

3.4.1 Simplifications. . . . . . . . . . . . . . 32

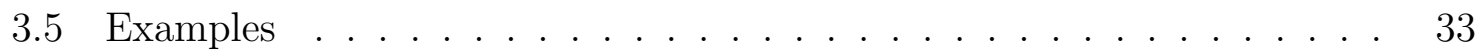

3.5.1 The Thue Morse substitution . . . . . . . . . . . . . . . . . . 33

3.5 .2 Penrose tilings . . . . . . . . . . . . . . . . . . 35

\begin{tabular}{ll}
\hline Concluding Questions & 39
\end{tabular}

$\begin{array}{ll}\text { References } & 43\end{array}$ 